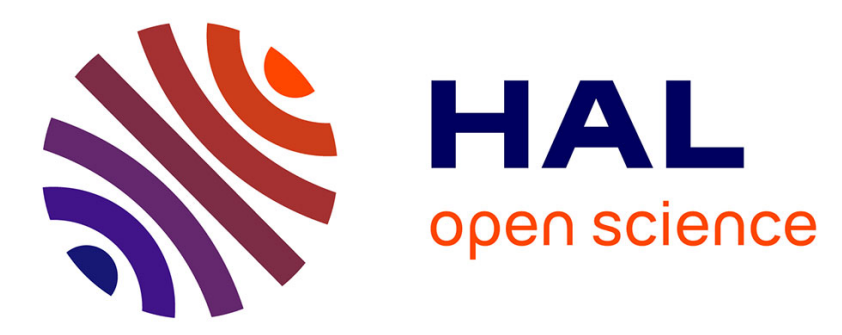

\title{
A simple computational homogenization method for structures made of heterogeneous linear viscoelastic materials
}

A. B. Tran, Julien Yvonnet, Qi-Chang He, C. Toulemonde, J. Sanahuja

\section{To cite this version:}

A. B. Tran, Julien Yvonnet, Qi-Chang He, C. Toulemonde, J. Sanahuja. A simple computational homogenization method for structures made of heterogeneous linear viscoelastic materials. Computer Methods in Applied Mechanics and Engineering, 2011, 200 (45-46), pp.2956-2970. 10.1016/j.cma.2011.06.012 . hal-00725389

\section{HAL Id: hal-00725389 \\ https://hal.science/hal-00725389}

Submitted on 10 May 2013

HAL is a multi-disciplinary open access archive for the deposit and dissemination of scientific research documents, whether they are published or not. The documents may come from teaching and research institutions in France or abroad, or from public or private research centers.
L'archive ouverte pluridisciplinaire HAL, est destinée au dépôt et à la diffusion de documents scientifiques de niveau recherche, publiés ou non, émanant des établissements d'enseignement et de recherche français ou étrangers, des laboratoires publics ou privés. 


\title{
A simple computational homogenization method for structures made of linear heterogeneous viscoelastic materials
}

\author{
A.B. $\operatorname{Tran}^{a}$, J. Yvonnet ${ }^{a, *}$, Q-C. $\mathrm{He}^{a}$, C. Toulemonde ${ }^{b}$, J. Sanahuja $^{b}$ \\ ${ }^{a}$ Université Paris-Est, Laboratoire Modélisation et Simulation Multi Échelle MSME \\ UMR 8208 CNRS, 5 bd Descartes, F-77454 Marne-la-Vallée, France. \\ ${ }^{b}$ EDF R\&D - Département MMC Site des Renardières - Avenue des Renardières - \\ Ecuelles, 77818 Moret sur Loing Cedex, France
}

\begin{abstract}
In this paper, a numerical multiscale method is proposed for computing the response of structures made of linearly non-aging viscoelastic and highly heterogeneous materials. In contrast with most of the approaches reported in the literature, the present one operates directly in the time domain and avoids both defining macroscopic internal variables and concurrent computations at micro and macro scales. The macroscopic constitutive law takes the form of a convolution integral containing an effective relaxation tensor. To numerically identify this tensor, a representative volume element (RVE) for the microstructure is first chosen. Relaxation tests are then numerically performed on the RVE. Correspondingly, the components of the effective relaxation tensor are determined and stored for different snapshots in time. At the macroscopic scale, a continuous representation of the effective relaxation tensor is obtained in the time domain by interpolating the data with the help of spline functions. The convolution integral characterizing the time-dependent macroscopic stress-strain relation is evaluated numerically. Arbitrary local linear viscoelastic laws and microstructure morphologies can be dealt with. Implicit algorithms are provided to compute the time-dependent response of a structure at the macroscopic scale by the finite element method. Accuracy and efficiency of the proposed approach are demonstrated through $2 \mathrm{D}$ and 3D numerical examples and applied to estimate the creep of structures made
\end{abstract}

\footnotetext{
${ }^{\mathrm{W}}$ Correspondance to J. Yvonnet

Email address: julien.yvonnet@univ-paris-est.fr ()
} 
of concrete.

Keywords: Computational homogenization, Linear viscoelasticity, Composites, Structures, Concrete

\section{Introduction}

Designing composite materials with tuned viscoelastic properties is a major concern in engineering. In polymer composites, damping properties can be desired in tandem with strength properties. In concrete structures, reducing the magnitude of creep allows diminution of the associated damage [16]. The related experimental relaxation tests are extremely costly and can last for months or years. Progresses in the design of high performance concrete then require predictive models and simulation methods taking into account the microstructure of the material.

Analytical methods for the homogenization of linear viscoelastic media have been proposed since the works of Hashin [7, 8], who exploited the correspondence principle between linear elasticity and viscoelasticity by mean of the Laplace transform. In the Laplace space, classical homogenization methods such as the self-consistent scheme [13, 25, 15, 1, 21, 2] and Mori-Tanaka technique $[26,6,18,5,3]$ can be applied. The main issue is then the inversion of the Laplace transform which, in most cases, need to be performed numerically (see e.g. $[27,9,14]$ ). Accuracy and computational costs of this numerical inversion are serious issues. When applied to homogenization, the restrictive assumptions underlying the analytical methods on the morphology and local constitutive laws prevent them from being applied to complex realistic microstructures. Then, numerical method must be employed to solve the microscale spatial equations. Some methodologies have been proposed. For example, in [17], the microscopic spatial equations are solved by the generalized cell method.

To overcome the limitations of approaches based on the Laplace transform, alternative numerical methods operating in the time domain have been suggested. Lahellec and Suquet [12] introduced a scheme in which the notion of macroscopic internal variables related to an effective viscous strain is involved. Their method is based on an incremental variational principle and the variational approach of Ponte-Castañeda [19]. Ricaud and Masson [20] proposed a different way taking advantage of the Prony-Dirichlet series expansion in the internal variable formulation. Another possible methodology 
corresponds to a two-scale numerical procedure $[11,4]$ where each integration point of the macroscopic structure is associated to a representative volume element and, at every time step, the macroscopic strains at each integration point are taken to be the boundary conditions for the relevant local problem. The numerical solution to this problem gives the effective stresses. These methodologies induce important computational costs due to the nested numerical solvers and the storage of internal variables, even though progresses have been made by means of parallel computing [4] or model reduction [29].

The purpose of this paper is to present an efficient and simple methodology to compute the effective time-dependent response of structures consisting of linearly viscoelastic heterogeneous materials and undergoing arbitrary loadings. The homogenized constitutive law of a linearly viscoelastic heterogeneous material takes the form of a convolution integral involving an effective relaxation tensor which cannot be in general determined analytically. One of the main steps of our approach is to numerically determine all the components of the effective relaxation tensor directly in the time domain. This is realized as follows: (i) a representative volume element (RVE) for the microstructure of the linearly viscoelastic heterogeneous material in question is chosen and subjected to appropriate relaxation test loadings; (ii) the overall time-dependent response of the RVE is computed by using some efficient algorithms (see e.g. [10, 22, 24]); (iii) the numerical results obtained at different time steps and stored during the previous preliminary computations are interpolated with some appropriate spline functions. Then, the convolution integral is evaluated numerically so as to yield the macroscopic stress-strain relation for the computation of structures.

Compared to existing approaches, the one elaborated in the present work offers the following advantages: (a) the method operates directly in the time domain and avoids the drawbacks of the techniques based on the Laplace transform; (b) the formulation needs not to introduce any macroscopic internal variables; (c) in contrast with the numerical methods using concurrent calculations at the microscopic and macroscopic scales, the data required to determine the effective constitutive laws can be calculated in a preliminary step, so that, once they are stored, structure calculations can be carried out without solving any new problems on the RVE (for a related work on nonlinear homogenization, see [28]); d) the implementation of the proposed approach is simple and classical implicit time-stepping algorithms can be directly employed. 


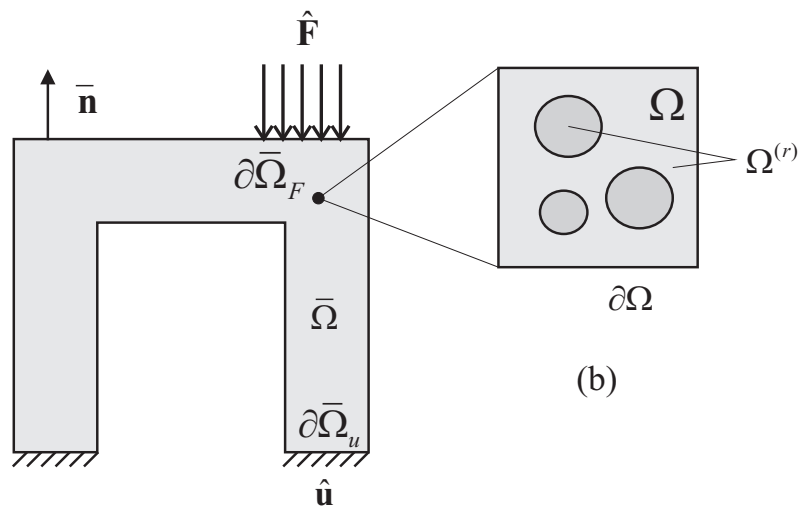

(a)

Figure 1: (a) Macroscopic structure and (b) Representative Volume Element

The paper is organized as follows. In the next section, we briefly review the equations and algorithms for formulating and solving the local viscoelastic problem defined over an RVE. In section 3 we present the methodology for sampling and interpolating the values of the effective relaxation tensor. Fully implicit algorithms are then detailed to compute the macroscopic structural response. In section 4, we illustrate the proposed method and test its accuracy and efficiency through different 2D and 3D examples, with applications to the analysis of structures made of concrete.

\section{Microscopic viscoelastic problem}

We consider a structure made of a heterogeneous material whose phases are linearly and non-aging viscoelastic. We assume that the microstructure is defined by a representative volume element occupying a domain $\Omega$, as depicted in figure $1 \mathrm{~b}$ ). The sub-domains occupied by the different phases are $\Omega^{(r)}(r=1,2, \ldots, R)$ such that $\Omega=\bigcup_{r=1}^{R} \Omega^{(r)}$. In this section, we review equations and algorithms for solving linear homogeneous viscoelastic problems. We focus on the generalized Maxwell model which, with an infinite number of branches, is the most general one for linear viscoelasticity.

\subsection{Linear viscoelasticity: generalized Maxwell model}

\subsection{1. $1 D$ formulation}

A linearly viscoelastic material can be characterized by a stress-strain relationship in the form of a convolution integral: 


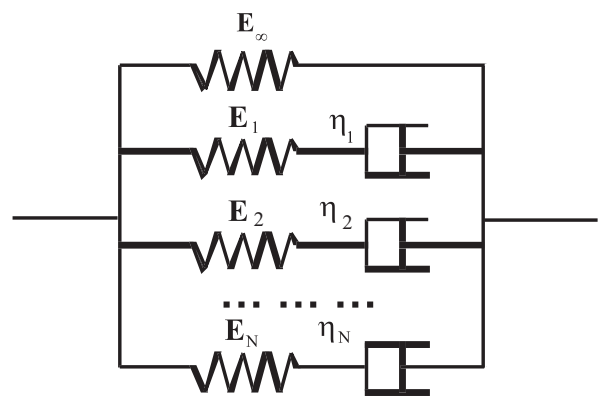

Figure 2: Schematic representation of the generalized Maxwell model.

$$
\sigma(t)=\int_{-\infty}^{t} G(t-s) \frac{d \varepsilon(s)}{d s} d s
$$

where $G(t)$ is the relaxation modulus function. The integral in (1) is a Riemann-Stieltjes integral. It will be convenient to consider only timedependent stress $\sigma(t)$ and strain $\varepsilon(t)$ which are null for $t<0$, and which may have jump discontinuities at $t=0$. In this case, we write (1) in the form

$$
\sigma(t)=\int_{0}^{t} G(t-s) \frac{d \varepsilon(s)}{d s} d s+G(t) \varepsilon(0) .
$$

We consider the generalized Maxwell model as depicted in figure 2. The corresponding relaxation modulus function is given by (see details in Appendix 7):

$$
G(t)=E_{\infty}+\sum_{i=1}^{N} E_{i} \exp \left(-t / \tau_{i}\right),
$$

where $N$ is the number of parallel viscoelastic elements, $E_{\infty}, E_{i}$ are Young's moduli as shown in figure 2 , and $\tau_{i}$ are the relaxation times of the parallel viscoelastic elements. Substituting (3) into (2), the total stress is given by

$$
\begin{gathered}
\sigma(t)=\int_{0}^{t} \dot{\sigma}_{\infty}(s) d s+\sum_{i=1}^{N} \int_{0}^{t} \gamma_{i} \exp \left(-(t-s) / \tau_{i}\right) \dot{\sigma}_{\infty}(s) d s \\
+\left(1+\sum_{i=1}^{N} \gamma_{i} \exp \left(-t / \tau_{i}\right)\right) \sigma_{\infty}(0),
\end{gathered}
$$


where $\sigma_{\infty}(t)=E_{\infty} \varepsilon(t)$ and $\gamma_{i}=E_{i} / E_{\infty}$. By introducing

$$
q_{i}=\int_{0}^{t} \gamma_{i} \exp [-(t-s)] / \tau_{i} \dot{\sigma}_{\infty}(s) d s
$$

as internal stress variables, we finally obtain

$$
\sigma(t)=\sum_{i=1}^{N} q_{i}+\sum_{i=1}^{N} \gamma_{i} \exp \left(-t / \tau_{i}\right) \sigma_{\infty}(0)+\sigma_{\infty}(t)
$$

\subsection{2. $3 D$ isotropic formulation}

In the three-dimensional (3D) isotropic case, the deviatoric and hydrostatic stresses are usually expressed separately. The stress-strain relationship of a linearly viscoelastic material is then given by:

$$
\left\{\begin{array}{l}
\operatorname{tr}(\boldsymbol{\sigma}(t))=\int_{0}^{t} G_{k}(t-s) \operatorname{tr}(\dot{\boldsymbol{\varepsilon}}(s)) d s+G_{k}(t) \operatorname{tr}(\boldsymbol{\varepsilon}(0)) \\
\operatorname{dev}(\boldsymbol{\sigma}(t))=\int_{0}^{t} G_{\mu}(t-s) \operatorname{dev}(\dot{\boldsymbol{\varepsilon}}(s)) d s+G_{\mu}(t) \operatorname{dev}(\boldsymbol{\varepsilon}(0))
\end{array}\right.
$$

where $\operatorname{tr}($.$) and \operatorname{dev}($.$) denote the trace and deviatoric parts of a tensor and$ $G_{k}(t)$ and $G_{\mu}(t)$ are the time-dependent shear and bulk moduli. For an isotropic compressible material described by the generalized Maxwell model, we have [22]:

$$
\left\{\begin{array}{l}
G_{k}(t)=3 k_{\infty}+\sum_{i=1}^{N} 3 k_{i}^{e} \exp \left(-t / \tau_{i}^{k}\right) \\
G_{\mu}(t)=2 \mu_{\infty}+\sum_{i=1}^{N} 2 \mu_{i}^{e} \exp \left(-t / \tau_{i}^{\mu}\right)
\end{array}\right.
$$

where $k_{\infty}$ and $\mu_{\infty}$ are the bulk and shear moduli of the elastic element, $k_{i}^{e}$ and $\mu_{i}^{e}$ are the elastic bulk and shear moduli of a viscoelastic element, say element $i$, and $\tau_{i}^{k}$ and $\tau_{i}^{\mu}$ are defined by

$$
\tau_{i}^{k}=\frac{k_{i}^{v}}{k_{i}^{e}} \quad \tau_{i}^{\mu}=\frac{\mu_{i}^{v}}{\mu_{i}^{e}}
$$

with $k_{i}^{v}$ and $\mu_{i}^{v}$ being the viscous bulk and shear moduli of viscoelastic element $i$. For later use, it is convenient to introduce the ratios

$$
\gamma_{i}^{k}=\frac{k_{i}^{e}}{k_{\infty}^{e}}, \quad \gamma_{i}^{\mu}=\frac{\mu_{i}^{e}}{\mu_{\infty}^{e}}
$$

In the present work, we will not consider any incompressible linear viscoelastic materials. 


\subsection{Strong form for the local problem}

In the following, we present the equations defined over an RVE and formulate the local viscoelastic problem that will be solved numerically. The solution to this problem will be used in the next section to construct the macroscopic constitutive law.

We consider $\Omega$ the RVE with boundary $\partial \Omega$ in figure $1 \mathrm{~b}$ ). Neglecting body forces, the equilibrium equations of the problem read

$$
\nabla \cdot \boldsymbol{\sigma}(t)=0 \text { in } \Omega
$$

while the time-dependent stress-strain relation can be written as

$$
\boldsymbol{\sigma}(t)=\mathcal{V}(t)\{\boldsymbol{\varepsilon}(t)\} .
$$

In (11), $\nabla \cdot($.$) is the divergence operator, \boldsymbol{\sigma}$ denotes the Cauchy stress tensor. In (12), the infinitesimal strain tensor is related to the displacement vector by $\boldsymbol{\varepsilon}(\mathbf{u})=\left(\nabla \mathbf{u}+\nabla \mathbf{u}^{T}\right) / 2$ and $\mathcal{V}(t)$ is the time-dependent linear operator associated to the viscoelastic model as expressed in Eqs. (7). Next, we prescribe the displacement boundary conditions as follows:

$$
\overline{\mathbf{u}}(t)=\overline{\boldsymbol{\varepsilon}}(t) \mathbf{x}+\tilde{\mathbf{u}} \text { on } \partial \Omega,
$$

where $\overline{\boldsymbol{\varepsilon}}(t)$ is a time-dependent macroscopic strain tensor, $\mathbf{x}$ is the position vector of a material point in $\Omega$ and $\tilde{\mathbf{u}}$ is a periodic displacement vector function. Eq. (13) corresponds to periodic boundary conditions. When the mesh is not periodic, as found for example in the 3D numerical example of section 4.3 , only the linear boundary conditions, namely $\tilde{\mathbf{u}}=0$, are prescribed.

\subsection{Discrete algorithm for the local viscoelastic problem}

To numerically solve the viscoelastic problem formulated above, a timestepping procedure is employed. The microscopic time interval $\mathcal{T}=\left[0, t^{\text {max }}\right]$ is discretized into time steps $t^{i}=(i-1) \Delta t$ with $i=1,2, \ldots, n, t^{\max }$ being the maximum simulation time and $\Delta t$ the microscopic time step assumed to be constant.

\subsubsection{Time-stepping}

For the 1D model, using equation (6), the stress at time $t^{n+1}$ is given by

$$
\sigma^{n+1}=\sum_{i=1}^{N} \gamma_{i} \exp \left(-t^{n+1} / \tau_{i}\right) \sigma_{\infty}^{(0)}+\sigma_{\infty}{ }^{n+1}+\sum_{i=1}^{N} q_{i}{ }^{n+1} .
$$


Splitting the exponential expression

$$
\exp \left(-\frac{t^{n+1}}{\tau_{i}}\right)=\exp \left(-\frac{t^{n}+\Delta t}{\tau_{i}}\right)=\exp \left(-\frac{t^{n}}{\tau_{i}}\right) \exp \left(-\frac{\Delta t}{\tau_{i}}\right)
$$

the internal variables can be written as

$$
\begin{aligned}
q_{i}^{n+1}= & \int_{0}^{t^{n+1}} \gamma_{i} \exp \left(-\left(t^{n+1}-s\right) / \tau_{i}\right) \dot{\sigma}_{\infty}(s) d s \\
= & \int_{0}^{t^{n}} \gamma_{i} \exp \left(-\left(t^{n+1}-s\right) / \tau_{i}\right) \dot{\sigma}_{\infty}(s) d s \\
& +\int_{t^{n}}^{t^{n+1}} \gamma_{i} \exp \left(-\left(t^{n+1}-s\right) / \tau_{i}\right) \dot{\sigma}_{\infty}(s) d s \\
= & \exp \left(-\Delta t / \tau_{i}\right) q_{i}{ }^{n} \\
& +\int_{t^{n}}^{t^{n+1}} \gamma_{i} \exp \left(-\left(t^{n+1}-s\right) / \tau_{i}\right) \dot{\sigma}_{\infty}(s) d s
\end{aligned}
$$

With the help of the approximation [24, 10, 22]:

$$
\dot{\sigma}_{\infty}(t) \simeq \frac{\sigma_{\infty}^{n+1}-\sigma_{\infty}^{n}}{\Delta t} \quad \text { for } \mathrm{t} \in\left[t^{n}, t^{n+1}\right],
$$

we obtain the recursive formula

$$
\begin{aligned}
q_{i}^{n+1}= & \exp \left(-\Delta t / \tau_{i}\right) q_{i}^{n} \\
& +\gamma_{i}\left[\frac{\sigma_{\infty}{ }^{n+1}-\sigma_{\infty}{ }^{n}}{\Delta t}\right] \int_{t^{n}}^{t^{n+1}} \exp \left(-\left(t^{n+1}-s\right) / \tau_{i}\right) d s \\
= & \exp \left(-\Delta t / \tau_{i}\right) q_{i}^{n} \\
& +\gamma_{i} \tau_{i} \frac{1-\exp \left(-\Delta t / \tau_{i}\right)}{\Delta t}\left[\sigma_{\infty}{ }^{n+1}-\sigma_{\infty}{ }^{n}\right] .
\end{aligned}
$$

For the 3D model, introducing (8) in (7) and using a time stepping yields at time $t^{n+1}$ :

$$
\left\{\begin{aligned}
\operatorname{tr}\left(\boldsymbol{\sigma}^{n+1}\right)= & \sum_{i=1}^{N} \gamma_{i}^{k} \exp \left(-t^{n+1} / \tau_{i}^{k}\right) \operatorname{tr}\left(\boldsymbol{\sigma}_{\infty}{ }^{(0)}\right) \\
& +\operatorname{tr}\left(\boldsymbol{\sigma}_{\infty}^{n+1}\right)+\sum_{i=1}^{N}\left(q_{i}^{k}\right)^{n+1} \\
\operatorname{dev}\left(\boldsymbol{\sigma}^{n+1}\right)= & \sum_{i=1}^{N} \gamma_{i}^{\mu} \exp \left(-t^{n+1} / \tau_{i}^{\mu}\right) \operatorname{dev}\left(\boldsymbol{\sigma}_{\infty}{ }^{(0)}\right) \\
& +\operatorname{dev}\left(\boldsymbol{\sigma}_{\infty}^{n+1}\right)+\sum_{i=1}^{N}\left(\mathbf{q}_{i}^{\mu}\right)^{n+1}
\end{aligned}\right.
$$


where $\sigma_{\infty}=\mathbb{C}_{\infty}^{e}: \varepsilon, \mathbb{C}_{\infty}^{e}$ being the stiffness tensor in absence of viscous effects. The internal variables can be calculated through a recursive formula $[22,24]$ :

$$
\begin{aligned}
\left(q_{i}^{k}\right)^{n+1}= & \exp \left(-\Delta t / \tau_{i}^{k}\right)\left(q_{i}^{k}\right)^{n} \\
& +\gamma_{i}^{k} \tau_{i}^{k} \frac{1-\exp \left(-\Delta t / \tau_{i}^{k}\right)}{\Delta t}\left(\operatorname{tr}\left(\boldsymbol{\sigma}_{\infty}{ }^{n+1}\right)-\operatorname{tr}\left(\boldsymbol{\sigma}_{\infty}{ }^{n}\right)\right) \\
\left(\mathbf{q}_{i}^{\mu}\right)^{n+1}= & \exp \left(-\Delta t / \tau_{i}^{\mu}\right)\left(\mathbf{q}_{i}^{\mu}\right)^{n} \\
& +\gamma_{i}^{\mu} \tau_{i}^{\mu} \frac{1-\exp \left(-\Delta t / \tau_{i}^{\mu}\right)}{\Delta t}\left(\operatorname{dev}\left(\boldsymbol{\sigma}_{\infty}{ }^{n+1}\right)-\operatorname{dev}\left(\boldsymbol{\sigma}_{\infty}{ }^{n}\right)\right)
\end{aligned}
$$

\subsubsection{Weak form and FEM implicit discretization}

The weak form associated with Eqs. (11-13) is given by: find $\mathbf{u}(t) \in \mathcal{D}=$ $\left\{\mathbf{u}(t)=\overline{\mathbf{u}}(t)\right.$ on $\left.\partial \Omega, \mathbf{u}(\mathrm{t}) \in \mathrm{H}^{1}(\Omega)\right\}$ such that

$$
\int_{\Omega} \boldsymbol{\sigma}(t): \boldsymbol{\varepsilon}(\delta \mathbf{u}) d \Omega=0 \quad \forall \delta \mathbf{u} \in H_{0}^{1}(\Omega)
$$

where $H_{0}^{1}(\Omega)=\left\{\delta \mathbf{u} \in H^{1}(\Omega), \delta \mathbf{u}=0\right.$ on $\left.\partial \Omega\right\}$ and $\overline{\mathbf{u}}(t)$ is a prescribed displacement according to Eq. (13). Employing an implicit time-stepping, equation (22) at time $t^{n+1}$ can be written as:

$$
\int_{\Omega} \boldsymbol{\sigma}^{n+1}: \boldsymbol{\varepsilon}(\delta \mathbf{u}) d \Omega=0
$$


Using the expressions for the deviatoric and hydrostatic parts of stress at $t^{n+1}(19)$, we obtain

$$
\begin{aligned}
\int_{\Omega} \boldsymbol{\sigma}^{n+1}: & \boldsymbol{\varepsilon}(\delta \mathbf{u}) d \Omega=\int_{\Omega}\left(\frac{1}{3} \operatorname{tr}\left(\boldsymbol{\sigma}^{n+1}\right) \mathbf{1}+\mathbf{d e v}\left(\boldsymbol{\sigma}^{n+1}\right)\right): \boldsymbol{\varepsilon}(\delta \mathbf{u}) d \Omega \\
= & \int_{\Omega}\left(\sum_{i=1}^{N} \gamma_{i}^{k} \exp \left(-t^{n+1} / \tau_{i}^{k}\right) \operatorname{tr}\left(\boldsymbol{\sigma}_{\infty}^{(0)}\right)\right) \frac{1}{3} \mathbf{1}: \boldsymbol{\varepsilon}(\delta \mathbf{u}) d \Omega \\
& +\int_{\Omega}\left(\operatorname{tr}\left(\boldsymbol{\sigma}_{\infty}^{n+1}\right)+\sum_{i=1}^{N}\left(q_{i}^{k}\right)^{n+1}\right) \frac{1}{3} \mathbf{1}: \boldsymbol{\varepsilon}(\delta \mathbf{u}) d \Omega \\
& +\int_{\Omega}\left(\sum_{i=1}^{N} \gamma_{i}^{\mu} \exp \left(-t^{n+1} / \tau_{i}^{\mu}\right) \operatorname{dev}\left(\boldsymbol{\sigma}_{\infty}^{(0)}\right)\right): \boldsymbol{\varepsilon}(\delta \mathbf{u}) d \Omega \\
& +\int_{\Omega}\left(\operatorname{dev}\left(\boldsymbol{\sigma}_{\infty}^{(n+1)}\right)+\sum_{i=1}^{N}\left(\mathbf{q}_{i}^{\mu}\right)^{n+1}\right): \boldsymbol{\varepsilon}(\delta \mathbf{u}) d \Omega .
\end{aligned}
$$

By introducing the recursive formula (20-21) into the above expression, it follows that

$$
\begin{aligned}
\int_{\Omega} \boldsymbol{\sigma}^{n+1}: & \boldsymbol{\varepsilon}(\delta \mathbf{u}) d \Omega=\int_{\Omega} \boldsymbol{\varepsilon}^{n+1}: \mathbb{C}^{n+1}: \boldsymbol{\varepsilon}(\delta \mathbf{u}) d \Omega \\
& +\int_{\Omega} \boldsymbol{\sigma}_{\infty}^{(0)}: \mathbb{I}_{1}: \boldsymbol{\varepsilon}(\delta \mathbf{u}) d \Omega \\
& +\int_{\Omega} \sum_{i=1}^{N}\left(\frac{1}{3} \mathbf{1} \chi_{i}^{k}\left(q_{i}^{k}\right)^{n}+\chi_{i}^{\mu}\left(\mathbf{q}_{i}^{\mu}\right)^{n}\right): \boldsymbol{\varepsilon}(\delta \mathbf{u}) d \Omega \\
& -\int_{\Omega} \boldsymbol{\sigma}_{\infty}^{n}: \mathbb{I}_{2}: \boldsymbol{\varepsilon}(\delta \mathbf{u}) d \Omega
\end{aligned}
$$

In this formula,

$$
\chi_{i}^{k}=\exp \left(-\Delta t / \tau_{i}^{k}\right), \quad \chi_{i}^{\mu}=\exp \left(-\Delta t / \tau_{i}^{\mu}\right),
$$

the tensors $\mathbb{C}^{n+1}, \mathbb{I}_{1}$ and $\mathbb{I}_{2}$ are defined by

$$
\begin{gathered}
\mathbb{C}^{n+1}=3 k_{\infty} M^{k} \mathbb{J}_{1}+2 \mu_{\infty} M^{\mu} \mathbb{J}_{2}, \\
\mathbb{I}_{1}=N^{k} \mathbb{J}_{1}+N^{\mu} \mathbb{J}_{2}, \quad \mathbb{I}_{2}=P^{k} \mathbb{J}_{1}+P^{\mu} \mathbb{J}_{2},
\end{gathered}
$$


where

$$
\mathbb{J}_{1}=\frac{1}{3} \mathbf{1} \otimes \mathbf{1}, \quad \mathbb{J}_{2}=\mathbb{I}-\frac{1}{3} \mathbf{1} \otimes \mathbf{1} .
$$

In (27)-(76), $(I)_{i j k l}=\frac{1}{2}\left(\delta_{i k} \delta_{j l}+\delta_{i l} \delta_{i k}\right)$ is the fourth-order identity tensor, $\mathbf{1}$ denotes the second-order unit tensor, and

$$
\begin{gathered}
M^{k}=1+\sum_{i=1}^{N} \gamma_{i}^{k} \tau_{i}^{k} \frac{1-\chi_{i}^{k}}{\Delta t}, \quad M^{\mu}=1+\sum_{i=1}^{N} \gamma_{i}^{\mu} \tau_{i}^{\mu} \frac{1-\chi_{i}^{\mu}}{\Delta t}, \\
P^{k}=\sum_{i=1}^{N} \gamma_{i}^{k} \tau_{i}^{k} \frac{1-\chi_{i}^{k}}{\Delta t}, \quad P^{\mu}=\sum_{i=1}^{N} \gamma_{i}^{\mu} \tau_{i}^{\mu} \frac{1-\chi_{i}^{\mu}}{\Delta t}, \\
N^{\mu}=\sum_{i=1}^{N} \gamma_{i}^{\mu} \exp \left(-t^{n+1} / \tau_{i}^{\mu}\right), \quad N^{k}=\sum_{i=1}^{N} \gamma_{i}^{k} \exp \left(-t^{n+1} / \tau_{i}^{k}\right) .
\end{gathered}
$$

By substituting the expression (25) into Eq. (23), we obtain the weak form:

$$
\begin{gathered}
\int_{\Omega} \boldsymbol{\varepsilon}\left(\mathbf{u}^{n+1}\right): \mathbb{C}^{n+1}: \boldsymbol{\varepsilon}(\delta \mathbf{u}) d \Omega=\int_{\Omega} \boldsymbol{\sigma}_{\infty}{ }^{n}: \mathbb{I}_{2}: \boldsymbol{\varepsilon}(\delta \mathbf{u}) d \Omega \\
-\int_{\Omega} \boldsymbol{\sigma}_{\infty}^{(0)}: \mathbb{I}_{1}: \boldsymbol{\varepsilon}(\delta \mathbf{u}) d \Omega-\int_{\Omega} \sum_{i=1}^{N}\left(\frac{1}{3} \mathbf{1} \chi_{i}^{k}\left(q_{i}^{k}\right)^{n}+\chi_{i}^{\mu}\left(\mathbf{q}_{i}^{\mu}\right)^{n}\right): \boldsymbol{\varepsilon}(\delta \mathbf{u}) d \Omega .
\end{gathered}
$$

The right-hand term of Eq. (33) can be calculated from the displacement solution given at time step $t^{n}$.

Applying a standard finite element discretization to the weak form (33) we obtain a discrete system of linear equations at time $t^{n+1}$ :

$$
\mathbf{K}^{n+1} \mathbf{d}^{n+1}=\mathbf{f}^{n+1}
$$

where $\mathbf{d}^{n+1}$ is the nodal displacement vector at time $t^{n+1}, \mathbf{K}^{n+1}$ and $\mathbf{f}^{n+1}$ are the global stiffness matrix and force vector, respectively. More precisely, the matrix $\mathbf{K}^{n+1}$ and vector $\mathbf{f}^{n+1}$ are provided by

$$
\mathbf{K}^{n+1}=\int_{\Omega} \mathbf{B}^{T} \mathbf{C}^{n+1} \mathbf{B} d \Omega
$$




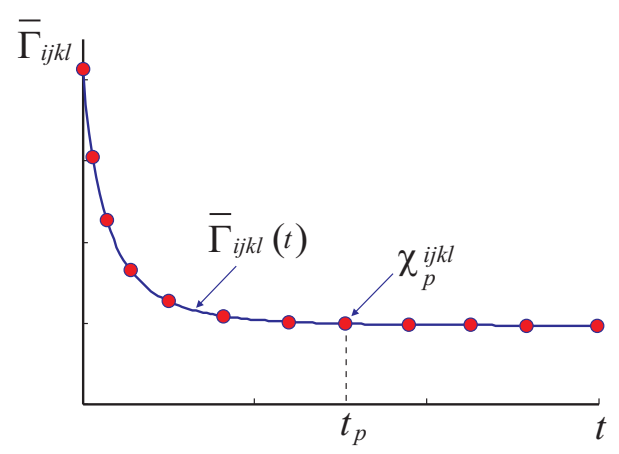

Figure 3: Discrete values of the macroscopic relaxation tensor $\chi_{p}^{i j k l}$ and continuous interpolation $\bar{\Gamma}_{i j k l}(t)$.

$$
\begin{gathered}
\mathbf{f}^{n+1}=-\int_{\Omega} \mathbf{B}^{T} \mathbf{I}_{1}\left[\boldsymbol{\sigma}_{\infty}^{0}\right] d \Omega \\
-\int_{\Omega} \sum_{i=1}^{N} \mathbf{B}^{T}\left(\frac{1}{3} \mathbf{I}_{1} \chi_{i}^{k}\left(q_{i}^{k}\right)^{n}+\chi_{i}^{\mu}\left(\mathbf{q}_{i}^{\mu}\right)^{n}\right) d \Omega+\int_{\Omega} \mathbf{B}^{T} \mathbf{I}_{2}\left[\boldsymbol{\sigma}_{\infty}^{n}\right] d \Omega
\end{gathered}
$$

where $\mathbf{B}$ and $\mathbf{N}$ are the matrices of shape functions derivatives and shape functions associated with the FEM approximation scheme and $\left[\boldsymbol{\sigma}_{\infty}^{n}\right]$ is the vector form related to the tensor $\boldsymbol{\sigma}_{\infty}^{n}$ while $\mathbf{C}^{n+1}, \mathbf{I}_{1}$ and $\mathbf{I}_{2}$ are the matrix forms associated with the fourth-order tensors $\mathbb{C}^{n+1}, \mathbb{I}_{1}$ and $\mathbb{I}_{2}$, respectively.

\section{Macroscopic model}

\subsection{A numerical homogenization model based on a numerical mapping}

The phases of the composite under investigation are assumed to be linearly viscoelastic and to have arbitrary morphology. Then, it can be shown that the effective, or macroscopic behavior of the composite remains linearly viscoelastic (see $[7,8]$ ) and is generally characterized by

$$
\begin{gathered}
\bar{\sigma}_{i j}(t)=\int_{-\infty}^{t} \bar{\Gamma}_{i j k l}(t-s) \frac{d \bar{\varepsilon}_{k l}(s)}{d s} d s \\
=\int_{0}^{t} \bar{\Gamma}_{i j k l}(t-s) \frac{d \bar{\varepsilon}_{k l}(s)}{d s} d s+\bar{\Gamma}_{i j k l}(t) \bar{\varepsilon}_{k l}(0),
\end{gathered}
$$


where $\bar{\sigma}_{i j}(t)=\left\langle\sigma_{i j}\right\rangle_{\Omega}$ and $\bar{\varepsilon}_{i j}(t)=\left\langle\varepsilon_{i j}\right\rangle_{\Omega}$ with $\langle.\rangle_{\Omega}$ denoting the volume average over $\Omega$. In Eq. (37), $\bar{\Gamma}_{i j k l}(t)$ are the components of the macroscopic relaxation tensor which is not known in closed form in the general case. In this work we seek to determine an approximated numerical expression for $\bar{\Gamma}_{i j k l}(t)$. More precisely, we introduce the numerically explicit mapping $\bar{\Gamma}_{i j k l}: \mathbb{R}^{+} \rightarrow \mathbb{R}$ defined by

$$
\bar{\Gamma}_{i j k l}(t) \simeq \sum_{p=1}^{M} \phi_{p}^{i j k l}(t) \chi_{p}^{i j k l},
$$

where $M$ is the number of non-zero shape functions at time $t$ and $\chi_{p}^{i j k l}$ are the components of the effective relaxation tensor function sampled at time $t_{p}$ (see figure 3) such that:

$$
\bar{\Gamma}_{i j k l}\left(t^{p}\right) \equiv \chi_{p}^{i j k l}
$$

and $\phi_{p}^{i j k l}(t)$ is the interpolation function related to the time step $t^{p}$.

Examples and choice of the shape functions will be discussed in the next section. The components $\chi_{p}^{i j k l}$ are the values of $\bar{\Gamma}_{i j k l}(t)$ computed numerically at the discrete time $t_{p}$. By choosing

$$
\bar{\varepsilon}(t)=H(t) \bar{\varepsilon}^{(i j)}
$$

where $H(t)$ is the Heaviside step function and $\overline{\boldsymbol{\varepsilon}}^{(i j)}$ is an elementary strain state, and by introducing (40) in (37), we obtain

$$
\bar{\sigma}_{i j}(t)=\int_{-\infty}^{t} \bar{\Gamma}_{i j k l}(t-s) \bar{\varepsilon}_{k l}^{(i j)} \delta(s) d s,
$$

with $\delta(t)$ being the Dirac delta function. With the help of the property

$$
\int_{-\infty}^{t} f(t-s) \delta(s) d s=f(t)
$$

we finally have

$$
\bar{\Gamma}_{i j k l}(t)=\frac{\bar{\sigma}_{i j}\left(t, \overline{\boldsymbol{\varepsilon}}^{(k l)}(t)\right)}{\bar{\varepsilon}_{0}}=\frac{\left\langle\sigma_{i j}\left(t, \overline{\boldsymbol{\varepsilon}}^{(k l)}(t)\right)\right\rangle}{\bar{\varepsilon}_{0}}
$$

where $\sigma_{i j}(t)$ is the stress field in the RVE obtained numerically by solving the problem (11)-(13), when $\bar{\varepsilon}(t)$ is given by (40) with 


$$
\overline{\boldsymbol{\varepsilon}}^{(k l)}=\frac{1}{2} \bar{\varepsilon}_{0}\left(\mathbf{e}_{k} \otimes \mathbf{e}_{l}+\mathbf{e}_{l} \otimes \mathbf{e}_{k}\right)
$$

In Eqs. (43)-(44), $\bar{\varepsilon}_{0}$ is an arbitrary constant, small enough to maintain the resulting microscopic and macroscopic strains small and such that no geometrical and mechanical nonlinearities occur.

\subsection{Algorithm for the macroscopic scale}

The macroscopic time interval $\overline{\mathcal{T}}=\left[0, \bar{t}^{\text {max }}\right]$ with $\bar{t}^{\text {max }}$ being the maximum simulation time is discretized into time steps $\bar{t}^{i}$, with $\bar{t}^{i}=(i-1) \bar{\Delta} t, i=$ $1,2, \ldots, \bar{n}$ and $\bar{\Delta} t$ the macroscopic time step taken to be constant. Note that $\bar{t}^{\max }$ and $\bar{\Delta} t$ may be different from $t^{\max }$ and $\Delta t$ used for the microscopic calculations.

We express the stress at time $\bar{t}^{n+1}$ by

$$
\begin{aligned}
\bar{\sigma}_{i j}{ }^{n+1} & =\int_{0}^{\bar{t}^{n+1}} \bar{\Gamma}_{i j k l}\left(\bar{t}^{n+1}-s\right) \frac{d \bar{\varepsilon}_{k l}(s)}{d s} d s+\bar{\Gamma}_{i j k l}\left(\bar{t}^{n+1}\right) \bar{\varepsilon}_{k l}^{(0)} \\
& =\sum_{m=0}^{n} \int_{\bar{t}^{m}}^{\bar{t}^{m+1}} \bar{\Gamma}_{i j k l}\left(\bar{t}^{n+1}-s\right) \frac{d \bar{\varepsilon}_{k l}(s)}{d s} d s+\bar{\Gamma}_{i j k l}\left(\bar{t}^{n+1}\right) \bar{\varepsilon}_{k l}^{(0)} .
\end{aligned}
$$

With the approximation

$$
\frac{d \bar{\varepsilon}_{k l}(t)}{d t} \simeq \frac{\bar{\varepsilon}_{k l}^{m+1}-\bar{\varepsilon}_{k l}^{m}}{\bar{\Delta} t}, \quad \text { for } \mathrm{t} \in\left[\bar{t}^{m}, \bar{t}^{m+1}\right]
$$

the stress at time $\bar{t}^{n+1}$ is given by

$$
\begin{gathered}
\bar{\sigma}_{i j}^{n+1}=\sum_{m=0}^{n}\left\{\left(\frac{\bar{\varepsilon}_{k l}^{m+1}-\bar{\varepsilon}_{k l}^{m}}{\bar{\Delta} t}\right) \int_{\bar{t}^{m}}^{\bar{t}^{m+1}} \bar{\Gamma}_{i j k l}\left(\bar{t}^{n+1}-s\right) d s\right\}+\bar{\Gamma}_{i j k l}\left(\bar{t}^{n+1}\right) \bar{\varepsilon}_{k l}^{(0)} \\
=\sum_{m=0}^{n}\left(\bar{\varepsilon}_{k l}^{m+1}-\bar{\varepsilon}_{k l}^{m}\right) A_{i j k l}^{(m, m+1)}\left(\bar{t}^{n+1}\right)+\bar{\Gamma}_{i j k l}\left(\bar{t}^{n+1}\right) \bar{\varepsilon}_{k l}^{(0)}
\end{gathered}
$$

where

$$
A_{i j k l}^{(m, m+1)}\left(\bar{t}^{n+1}\right)=\frac{1}{\bar{\Delta} t} \int_{\bar{t}^{m}}^{\bar{t}^{m+1}} \bar{\Gamma}_{i j k l}\left(\bar{t}^{n+1}-s\right) d s
$$




$$
=\frac{1}{\bar{\Delta} t} \sum_{p=1}^{M} \chi_{p}^{i j k l} \int_{\bar{t}^{m}}^{\bar{t}^{m+1}} \phi_{p}^{i j k l}\left(\bar{t}^{n+1}-s\right) d s .
$$

Remark that, in the above expression, the integral can be expressed in closed form if the shape functions $\phi_{p}^{i j k l}(t)$ are explicit analytical functions.

We now consider an open domain $\bar{\Omega} \subset \mathbb{R}^{3}$ with the external boundary $\partial \bar{\Omega}$ corresponding to the macroscopic domain (see figure $1 \mathrm{a}$ )) which is decomposed into two complementary and disjoint parts $\partial \bar{\Omega}_{u}$ and $\partial \bar{\Omega}_{F}$ where the Dirichlet and Neumann boundary conditions are prescribed, respectively. At time $\bar{t}^{n+1}$, we have

$$
\begin{aligned}
& \nabla \cdot \overline{\boldsymbol{\sigma}}^{n+1}+\mathbf{b}=0 \text { in } \bar{\Omega}, \\
& \overline{\boldsymbol{\sigma}}^{n+1} \mathbf{n}=\overline{\mathbf{f}}^{n+1} \text { on } \partial \bar{\Omega}_{F}, \\
& \overline{\mathbf{u}}^{n+1}=\overline{\mathbf{v}}^{n+1} \text { on } \partial \bar{\Omega}_{u},
\end{aligned}
$$

where $\mathbf{b}$ is body force, $\mathbf{n}$ is the unit outward normal vector to $\partial \Omega, \overline{\mathbf{f}}^{n+1}$ and $\overline{\mathbf{v}}^{n+1}$ are prescribed forces and displacements at time $\bar{t}^{n+1}$, respectively. Let $\overline{\mathbf{u}}^{n+1}$ be the macroscopic displacement vector of a point in $\bar{\Omega}$. The weak form associated with equations (49-51) is given as follows:

Find $\overline{\mathbf{u}}^{n+1}, \overline{\mathbf{u}}^{n+1}=\overline{\mathbf{v}}^{n+1}$ on $\partial \bar{\Omega}_{u}$ and $\overline{\mathbf{u}}^{n+1} \in H^{1}(\bar{\Omega})$ such that

$$
\int_{\bar{\Omega}} \overline{\boldsymbol{\sigma}}^{n+1}: \overline{\boldsymbol{\varepsilon}}(\delta \mathbf{u}) d \bar{\Omega}=\int_{\bar{\Omega}} \mathbf{b} \cdot \delta \mathbf{u} d \bar{\Omega}+\int_{\partial \bar{\Omega}_{F}} \overline{\mathbf{f}}^{n+1} \cdot \delta \mathbf{u} d \bar{\Gamma}
$$

$\forall \delta \mathbf{u} \in H_{0}^{1}(\bar{\Omega})$ and $\delta \mathbf{u}=\mathbf{0}$ on $\partial \bar{\Omega}_{u}$.

Inserting (47) into (52) and setting $\delta \bar{\varepsilon}_{i j}=[\overline{\boldsymbol{\varepsilon}}(\delta \mathbf{u})]_{i j}$, it follows that

$$
\begin{gathered}
\int_{\bar{\Omega}} \delta \bar{\varepsilon}_{i j} A_{i j k l}^{(n, n+1)}\left(\bar{t}^{n+1}\right) \bar{\varepsilon}_{k l}^{n+1} d \bar{\Omega}=\int_{\bar{\Omega}} \delta u_{i} b_{i} d \bar{\Omega}+\int_{\partial \bar{\Omega}_{F}} \delta u_{i} \bar{f}_{i}^{n+1} d \bar{\Gamma} \\
\quad-\sum_{m=0}^{n-1} \int_{\bar{\Omega}} \delta \bar{\varepsilon}_{i j} A_{i j k l}^{(m, m+1)}\left(\bar{t}^{n+1}\right)\left(\bar{\varepsilon}_{k l}^{m+1}-\bar{\varepsilon}_{k l}^{m}\right) d \bar{\Omega} \\
+\int_{\bar{\Omega}} \delta \bar{\varepsilon}_{i j} A_{i j k l}^{(n, n+1)}\left(\bar{t}^{n+1}\right) \bar{\varepsilon}_{k l}^{n} d \bar{\Omega}-\int_{\bar{\Omega}} \delta \bar{\varepsilon}_{i j} \bar{\Gamma}_{i j k l}\left(\bar{t}^{n+1}\right) \bar{\varepsilon}_{k l}^{(0)} d \bar{\Omega}
\end{gathered}
$$


Introducing a standard finite element approximation and owing to the arbitrariness of the variations, we obtain at time $\bar{t}^{n+1}$ a system of linear equations:

$$
\overline{\mathbf{K}}^{n+1} \overline{\mathbf{u}}^{n+1}=\overline{\mathbf{f}}_{e x t}^{n+1}-\overline{\mathbf{f}}_{V}^{n+1},
$$

with

$$
\begin{gathered}
\overline{\mathbf{K}}^{n+1}=\int_{\bar{\Omega}} \mathbf{B}^{T}[\mathbf{A}]^{(n, n+1)} \mathbf{B} d \bar{\Omega} \\
\overline{\mathbf{f}}_{e x t}=\int_{\bar{\Omega}} \mathbf{N}^{T} \mathbf{b} d \bar{\Omega}+\int_{\partial \bar{\Omega}_{F}} \mathbf{N}^{T} \overline{\mathbf{f}}^{n+1} d \bar{\Gamma} \\
\overline{\mathbf{f}}_{V}^{n+1}=\sum_{m=0}^{n-1} \int_{\bar{\Omega}} \mathbf{B}^{T}\left[\mathbf{A}^{(m, m+1)}\left(\bar{t}^{n+1}\right)\right]\left(\left[\bar{\varepsilon}^{m+1}\right]-\left[\bar{\varepsilon}^{m}\right]\right) d \bar{\Omega} \\
-\int_{\bar{\Omega}} \mathbf{B}^{T}\left[\mathbf{A}^{(n, n+1)}\left(\bar{t}^{n+1}\right)\right]\left[\bar{\varepsilon}^{n}\right] d \bar{\Omega}+\int_{\bar{\Omega}} \mathbf{B}^{T}\left[\boldsymbol{\Gamma}\left(\bar{t}^{n+1}\right)\right]\left[\bar{\varepsilon}^{(0)}\right] d \bar{\Omega} .
\end{gathered}
$$

Above, $\left[\mathbf{A}^{(m, m+1)}\left(\bar{t}^{n+1}\right)\right],\left[\boldsymbol{\Gamma}\left(\bar{t}^{n+1}\right)\right]$ are the matrix forms of the fourth-order tensors $A_{i j k l}^{(m, m+1)}\left(\bar{t}^{n+1}\right)$ and $\bar{\Gamma}_{i j k l}\left(\bar{t}^{n+1}\right)$, and $\left[\overline{\boldsymbol{\varepsilon}}^{n}\right]$ is the vector form of the second-order tensor $\bar{\varepsilon}^{n}$.

We notice that the vector $\overline{\mathbf{f}}_{V}^{n+1}$ in equation (57) depends on $\bar{\varepsilon}^{(0)}, \bar{\varepsilon}^{1}, \ldots$, $\bar{\varepsilon}^{n}$. As opposed to recursive algorithms $[22,24]$ it is here necessary to store macroscopic strains history in all elements of the macroscopic domain, for every time step. Memory limitations may appear when the structure mesh is very fine and the macroscopic time step is small. This point would deserve further improvement in the future, even though the technique remains still far less expensive than other direct approaches like multilevel numerical methods $[4,11]$, as will be shown in section 4 .

\subsection{Choice of interpolation and extrapolation functions}

Different choices are possible for interpolation functions $\phi_{p}^{i j k l}(t)$. In this paper, cubic spline functions are adopted. For $t>t^{\max }$, the continuous representation of $\bar{\Gamma}_{i j k l}(t)$ is obtained by extrapolation, accounting for asymptotic properties of $\bar{\Gamma}_{i j k l}(t)$. In figure 4 , the continuous curve illustrates the interpolation part of the continuous representation whereas the dashed curve represents the extrapolation part. The spline functions $f_{\text {in }}(t)=\left\{f_{\text {in }}^{(i)}(t)\right.$ if $\left.t \in\left[t^{i-1}, t^{i}\right]\right\}$, 


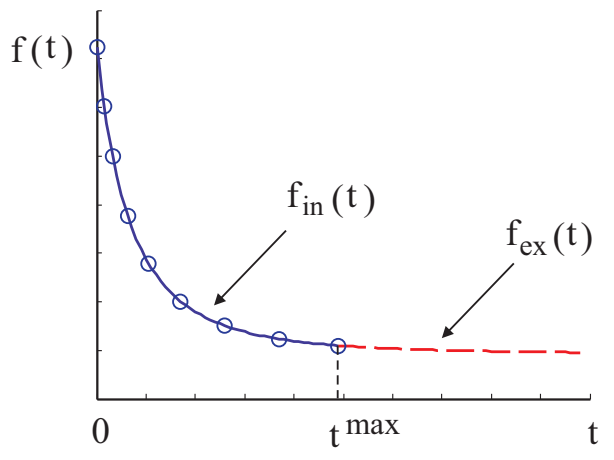

Figure 4: Extrapolation of the effective relaxation tensor components for $t>t^{\max }$.

are widely used to construct interpolations from discrete values. Each shape function $f_{i n}^{(i)}$ is a cubic polynomial and twice continuously differentiable, whose coefficients are determined by the requirement of verifying at each snapshot $t_{i}$ the following equations:

$$
\left\{\begin{aligned}
f_{i n}^{(i)}\left(t_{i}\right) & =f_{i n}^{(i+1)}\left(t_{i}\right), \\
f_{i n}^{(i) \prime}\left(t_{i}\right) & =f_{i n}^{(i+1) \prime}\left(t_{i}\right), \\
f_{i n}^{(i) \prime \prime}\left(t_{i}\right) & =f_{i n}^{(i+1) \prime \prime}\left(t_{i}\right) .
\end{aligned}\right.
$$

In the present work, the Matlab @ Spline Toolbox with functions "spline.m" and "ppval.m" are used to compute the aforementioned coefficients. The spline shape functions have a high accuracy for smooth curves, allowing a reduced number of sampling points. Since the relaxation tensor components present in general no sharp variations by nature, spline functions are an interesting choice.

As only a finite number of snapshots can be calculated, it is necessary to define an extrapolation procedure to compute values of the relaxation tensor after the last snapshot at the time step $t^{\max }$. Due to the fact that the relaxation tensor components rate vanish for $t \rightarrow \infty$, we define $\bar{\Gamma}_{i j k l}(t)$ in the interval $t \in\left[t^{\max },+\infty[\right.$ by

$$
f_{e x}(t)=a e^{-b t}+c
$$

which has the property that $f_{e x}(t \rightarrow \infty)=c$. Parameters $a, b$ and $c$ are 
determined by continuity conditions at time step $t^{\max }$ :

$$
\left\{\begin{array} { l } 
{ f _ { e x } ( t ^ { \operatorname { m a x } } ) = f _ { i n } ( t ^ { \operatorname { m a x } } ) } \\
{ f _ { e x } ^ { \prime } ( t ^ { \operatorname { m a x } } ) = f _ { i n } ^ { \prime } ( t ^ { \operatorname { m a x } } ) } \\
{ f _ { e x } ^ { \prime \prime } ( t ^ { \operatorname { m a x } } ) = f _ { i n } ^ { \prime \prime } ( t ^ { \operatorname { m a x } } ) }
\end{array} \Leftrightarrow \left\{\begin{array}{l}
a e^{-b t^{\max }+c}=f_{i n}\left(t^{\max }\right) \\
-a b e^{-b t^{\max }}=f_{i n}^{\prime}\left(t^{\max }\right) \\
a b^{2} e^{-b t^{\max }}=f_{i n}^{\prime \prime}\left(t^{\max }\right)
\end{array}\right.\right.
$$

The solution to (60) is given by

$$
\begin{aligned}
& b=-\frac{f_{i n}^{\prime \prime}\left(t^{\max }\right)}{f_{i n}^{\prime}\left(t^{\max }\right)}, \\
& a=-\frac{f_{i n}^{\prime}\left(t^{\max }\right)}{b e^{-b t^{\max }}}, \\
& c=f_{\text {in }}\left(t^{\max }\right)-a e^{-b t^{\max }} .
\end{aligned}
$$

It is worth noting that $c$ can be determined directly by computing the effective linear elastic properties of the material. For stationary regime, we have $c=C_{i j k l}^{\infty}$. In this case, the third equation of (60) is removed, $a$ and $b$ have the expressions

$$
\begin{aligned}
& b=-\frac{f_{\text {in }}^{\prime}\left(t^{\max }\right)}{f_{\text {in }}\left(t^{\max }\right)-c}, \\
& a=\frac{f_{\text {in }}\left(t^{\text {max }}\right)-c}{e^{-b\left(t^{\max }\right)}} .
\end{aligned}
$$

\subsection{Summary of the multiscale algorithm}

It is useful to summarize the main steps of the proposed multiscale procedure. First, calculations are carried out on the RVE at the microscale. Once the discrete values of the effective relaxation tensor $\chi_{p}^{i j k l}$ are obtained, calculations can be done for structures at the macroscopic scale without performing new microscopic computations.

\subsubsection{Microscale calculations}

The microscopic step aims to determine the discrete values of the effective relaxation tensor $\chi_{p}^{i j k l}$. According to equation (40), we apply three elementary macroscopic strains states $\overline{\boldsymbol{\varepsilon}}^{(i j)}$ in 2D and six elementary macroscopic strains states in 3D. For example, $\overline{\boldsymbol{\varepsilon}}^{(i j)}$ in 2D are given by 
$\overline{\boldsymbol{\varepsilon}}^{(11)}=\bar{\varepsilon}_{0}\left(\begin{array}{lll}1 & 0 & 0 \\ 0 & 0 & 0 \\ 0 & 0 & 0\end{array}\right) ; \overline{\boldsymbol{\varepsilon}}^{(22)}=\bar{\varepsilon}_{0}\left(\begin{array}{lll}0 & 0 & 0 \\ 0 & 1 & 0 \\ 0 & 0 & 0\end{array}\right) ; \overline{\boldsymbol{\varepsilon}}^{(12)}=\bar{\varepsilon}_{0}\left(\begin{array}{ccc}0 & 1 / 2 & 0 \\ 1 / 2 & 0 & 0 \\ 0 & 0 & 0\end{array}\right)$

with $\bar{\varepsilon}_{0}=10^{-3}$. In eq. (61), the strains remain within the small strain range. For each elementary strain, we solve the problem (11-12-13) numerically by (34) for $t=\left\{t^{0}, t^{1}, \ldots, t^{n}\right\}$ and compute $\bar{\sigma}_{i j}\left(t^{q}\right)=\left\langle\sigma_{i j}\left(t^{q}, \mathbf{x}\right)\right\rangle_{\Omega}$. The relaxation tensor defined in Eq. (43) is finally provided by

$$
\bar{\Gamma}_{i j k l}\left(t^{q}\right)=\chi_{q}^{i j k l}=\frac{\bar{\sigma}_{i j}\left(t^{q}, \overline{\boldsymbol{\varepsilon}}^{(k l)}\right)}{\bar{\varepsilon}_{0}}, \quad \mathrm{q}=1,2, \ldots, \mathrm{n},
$$

where $\overline{\boldsymbol{\varepsilon}}=H(t) \overline{\boldsymbol{\varepsilon}}^{(k l)}$ in the expression of boundary conditions (13) .

We then store all numerical values of $\chi_{q}^{i j k l}$ for the macroscopic scale calculations.

\subsubsection{Macroscale calculations}

The step-by-step algorithm for the macroscale can be summarized as follows:

WHILE $t<T$

1. At time $\bar{t}^{n+1}, \bar{\varepsilon}_{k l}^{q}, q=1,2, \ldots, \bar{n}$ are given at each integration point.

LOOP over integration points in the macroscopic mesh

(a) Compute the elementary matrix $\overline{\mathbf{K}}^{e, n+1}$ and the elementary vector $\overline{\mathbf{f}}_{\text {ext }}^{e}$ using (55)-(56).

(b) Compute the elementary vector $\overline{\mathbf{f}}_{v}^{e, n+1}$ using (57).

(c) Assemble $\overline{\mathbf{K}}^{e, n+1}, \overline{\mathbf{f}}_{e x t}^{e}$ and $\overline{\mathbf{f}}_{v}^{e, n+1}$.

\section{END}

2. Solve the system of linear equations (54).

3. Compute and store $\bar{\varepsilon}_{k l}^{n+1}$ for all integration points of the macroscopic domain.

4. Go to step 1.

\section{END}




\subsection{Remarks}

The class of boundary loads and body forces for which the proposed approximation is reasonable is ultimately delimited by the assumption of linear non-aging viscoelasticity and by the scale separation requirement underlying the proposed computational homogenization method. Precisely, the class of boundary loadings and body forces for which the proposed approach is suitable can be defined as follows:

1. The amplitudes of boundary loadings and body forces complying with the assumption of linear viscoelasticity must be such that the resulting microscopic and macroscopic strains are small and no geometrical and mechanical nonlinearities occur.

2. The frequencies of boundary loadings and body forces compatible with the scale separation requirement must be such that the typical length scale of the inhomogeneities in a representative volume element is small with respect to the typical wave length of the boundary loadings and body forces.

3. In the case of oscillatory loads, special numerical treatments are in general necessary for a high frequency due to the implicit time-stepping procedure at the macroscopic scale described in section 3.2. As the method operates in the time domain, a high frequency may entail very small time steps at the macroscopic scale. This issue is however not specific to the present method but to any numerical procedure operating in the time domain.

\section{Numerical examples}

In this section, the accuracy, efficiency and memory requirements of the method elaborated in the present paper are tested. First, one-scale calculations are performed to test the results of the proposed homogenization procedure for some arbitrary time-dependent loadings applied to the RVE. Second, two-scale examples in 2D and 3D are presented. Two different RVE with very distinct morphological characteristics are studied, as depicted in figure 5: an RVE with a single elliptical inclusion yielding an effective anisotropic behavior and an RVE with many randomly distributed inclusions leading to an isotropic effective behavior. The constitutive laws of the phases are taken to comply with the generalized Maxwell model. The numerical values of the relevant material parameters will be specified in each example. 


\subsection{One scale numerical tests}

\subsubsection{RVE containing an elliptical inclusion}

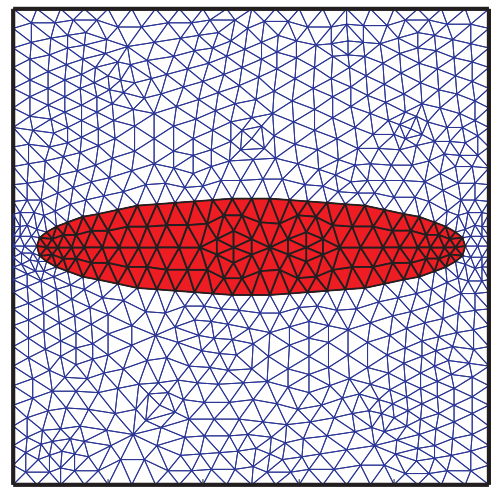

a)

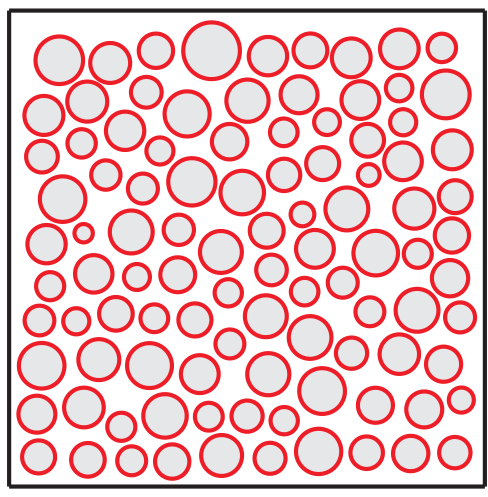

b)

Figure 5: a) Mesh and geometry of the RVE containing one elliptical inclusion; b) Geometry of the RVE containing 100 circular inclusions.

The RVE of figure 5 a) consists of an elliptical inclusion embedded in a unit square domain, the semi axes of the ellipse being equal to $r_{a}=0.45$ and $r_{b}=0.1$. The objective of this first test is to compare the effective response of the RVE computed through the proposed method with the one obtained by directly employing FEM. A conforming mesh of 1264 linear triangles is used. The material forming the matrix is linearly viscoelastic and isotropic while the inclusion is linearly elastic and isotropic. More precisely, the matrix is described by a generalized Maxwell model with one elastic branch and 5 spring-dampers branches (see figure 2). The numerical values of the corresponding material parameters are given in Table 1, where the indices $i$ and $m$ refer to the inclusion and matrix, respectively.

We apply the procedure described in section 3.1 so as to compute the macroscopic relaxation tensor. Some components of the latter are depicted in figure 6 a).

Next, we impose strain $\bar{\varepsilon}(t)$ on the boundary of the RVE and compute $\bar{\sigma}_{i j}(t)$ by using the FEM and proposed method. Periodic boundary conditions (13) are prescribed by means of Lagrange multipliers. The results are compared and presented in figure 8. In this figure, we have $\bar{\varepsilon}(t)=F(t) \bar{\varepsilon}^{A}$ with $\bar{\varepsilon}^{A}$ given by 
Table 1: Material parameters of viscoelastic phases in RVE of example 4.1.1.

\begin{tabular}{llllll}
\hline Matrix parameters & & & & & \\
\hline$E_{\infty, m}$ (MPa.days) & 13909 & & & & \\
$\nu_{\infty, m}$ & 0.256 & & & & \\
$E_{m}^{e}$ (MPa.days) & 231 & 322 & 425 & 630 & 577 \\
$\nu_{m}^{e}$ & 0.1 & 0.2 & 0.3 & 0.1 & 0.25 \\
$E_{m}^{v}$ (MPa.days) & 201200 & 255500 & 348900 & 503000 & 657700 \\
$\nu_{m}^{v}$ & 0.1 & 0.2 & 0.3 & 0.1 & 0.25 \\
\hline Inclusion parameters & & & & & \\
\hline$E_{i}$ (MPa) & 2398400 & & & & \\
$\nu_{i}$ & 0.28 & & & & \\
\hline
\end{tabular}

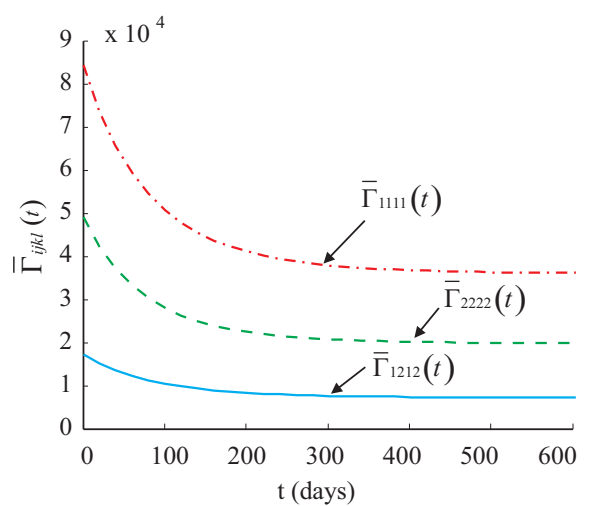

a)

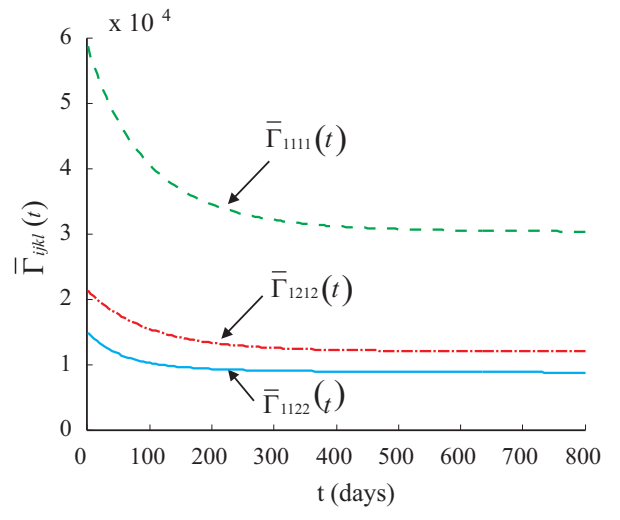

b)

Figure 6: Some components of the macroscopic relaxation tensor for a) the RVE containing one elliptical inclusion; b) the RVE containing 100 inclusions. 


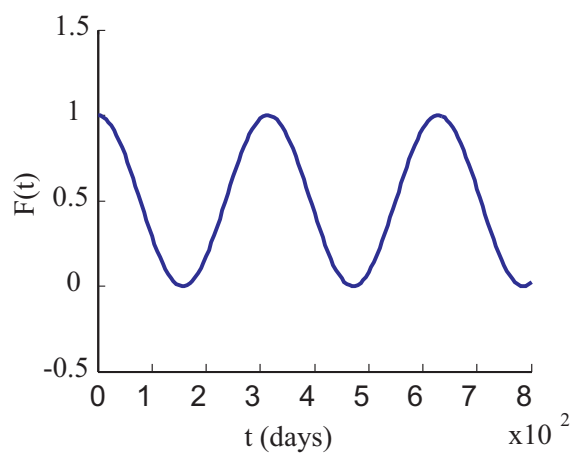

a)

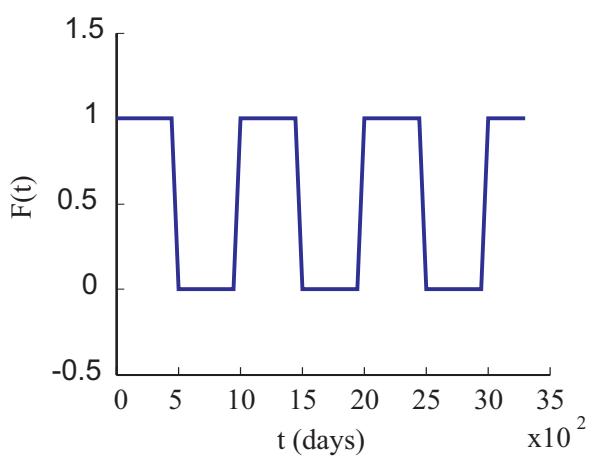

b)

Figure 7: Two types of time-dependent loading functions $F(t)$ : a) $F(t)=\frac{1}{2}\left(1+\cos \left(\frac{t}{50}\right)\right)$, b) $F(t)=\frac{1}{2}\left(1+(-1)^{\left\lfloor\frac{t}{500}\right\rfloor}\right)$.

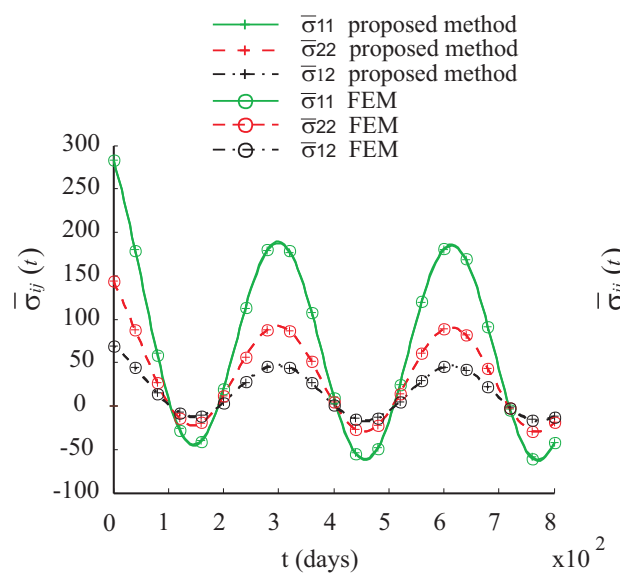

a)

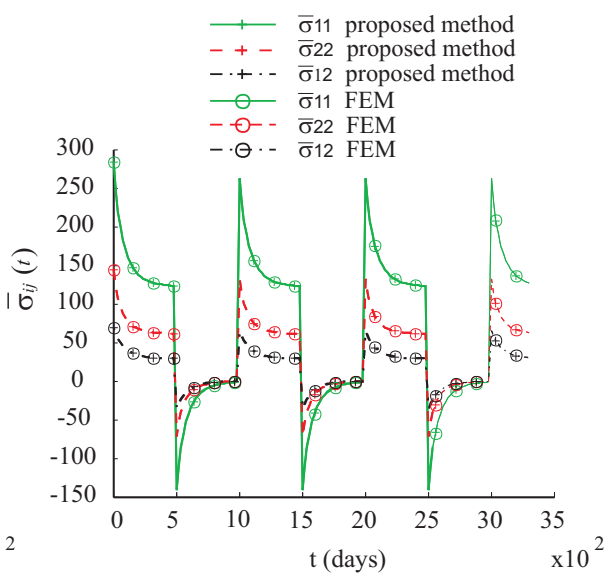

b)

Figure 8: Comparison between the proposed method and a direct FEM calculation for the test of RVE containing one elliptical inclusion: a) $F(t)=\frac{1}{2}\left(1+\cos \left(\frac{t}{50}\right)\right)$; b) $F(t)=$ $\frac{1}{2}\left(1+(-1)^{\left\lfloor\frac{t}{500}\right\rfloor}\right)$. 


$$
\overline{\boldsymbol{\varepsilon}}^{A}=\left(\begin{array}{lll}
3 & 2 & 0 \\
2 & 2 & 0 \\
0 & 0 & 0
\end{array}\right) 10^{-3}
$$

and $F(t)$ taken to be first the time-dependent sinusoidal function

$$
F(t)=\frac{1}{2}\left(1+\cos \left(\frac{t}{500}\right)\right)
$$

and then the function

$$
F(t)=\frac{1}{2}\left(1+(-1)^{\left\lfloor\frac{t}{500}\right\rfloor}\right)
$$

where $\lfloor x\rfloor$ denotes the floor function, i.e. the greatest integer less than or equal to $x$. The function $F(t)$ is plotted in figure 7 . We observe a very good agreement between the solution given by our approach and the reference (direct FEM) solution in each case.

\subsubsection{RVE containing 100 circular inclusions}

The objective of this example is to demonstrate the capability of the method to handle complex microstructures. Here we consider the RVE of figure $5 \mathrm{~b}$ ) containing 100 inclusions embedded in a unit square domain. The radii of the inclusions are randomly generated with a uniform probability distribution between $r_{\min }=0.0193$ and $r_{\max }=0.0595$. The volume fraction of inclusions is $f=0.4425$. The positions of inclusions centers are randomly generated with a uniform probability law and a non penetration constraint. In this example, both the matrix and inclusion phases are linearly viscoelastic and isotropic. The matrix is characterized by the Maxwell generalized model with an elastic branch and 5 spring-dampers, and the inclusions are described by the one with 4 spring-dampers (see figure 2 ). The numerical values of the material parameters are indicated in table 2 .

The RVE is meshed with 109948 linear triangular elements. Some components of the macroscopic relaxation tensor are shown in figure $6 \mathrm{~b}$ ). As in the previous test, different time-dependent loadings are prescribed with

$$
\bar{\varepsilon}^{A}=\left(\begin{array}{lll}
4 & 2 & 0 \\
2 & 6 & 0 \\
0 & 0 & 0
\end{array}\right) 10^{-3} .
$$

Figure 9 shows the results given by our method and a direct FEM. Again, very good agreement is observed for each choice of $F(t)$. 
Table 2: Material parameters of viscoelastic phases in RVE of example 4.1.2.

\begin{tabular}{llllll}
\hline Matrix parameters & & & & & \\
\hline$E_{\infty, m}$ (MPa.days) & 13909 & & & & \\
$\nu_{\infty, m}$ & 0.256 & & & & \\
$E_{m}^{e}$ (MPa.days) & 2310 & 3220 & 4250 & 6300 & 5770 \\
$\nu_{m}^{e}$ & 0.1 & 0.2 & 0.3 & 0.1 & 0.25 \\
$E_{m}^{v}$ (MPa.days) & 201200 & 255500 & 348900 & 503000 & 657700 \\
$\nu_{m}^{v}$ & 0.1 & 0.2 & 0.3 & 0.1 & 0.25 \\
\hline Inclusion parameters & & & & & \\
\hline$E_{i}^{e}(\mathrm{MPa})$ & 89000 & & & & \\
$\nu_{i}^{e}$ & 0.15 & & & & \\
$E_{i}^{e}(\mathrm{MPa})$ & 584 & 689 & 752 & 880 & \\
$\nu_{i}^{e}$ & 0.12 & 0.25 & 0.32 & 0.18 & \\
$E_{i}^{v}(\mathrm{MPa})$ & 60000 & 105000 & 144000 & 186000 & \\
$\nu_{i}^{v}$ & 0.2 & 0.12 & 0.1 & 0.22 & \\
\hline
\end{tabular}

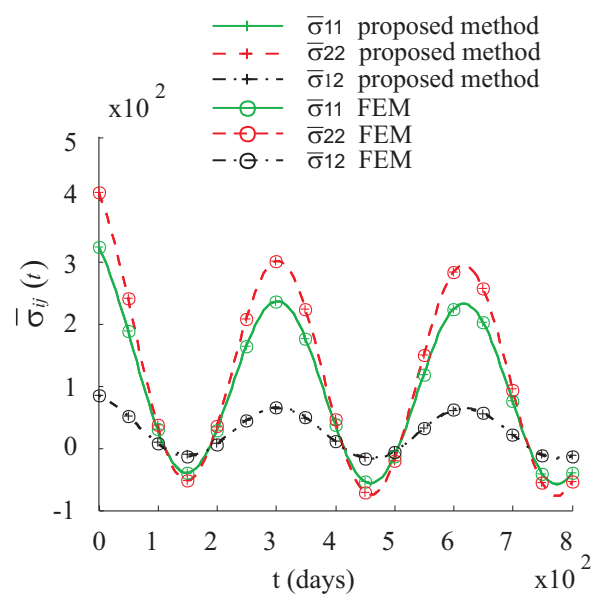

a)

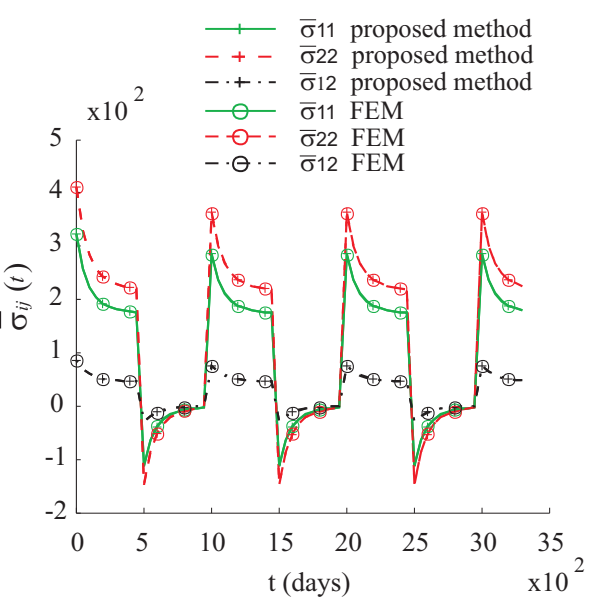

b)

Figure 9: Comparison between the proposed method and a direct FEM calculation for the test of RVE containing 100 inclusions: a) $F(t)=\frac{1}{2}\left(1+\cos \left(\frac{t}{50}\right)\right)$; b) $F(t)=\frac{1}{2}(1+$ $(-1)^{\left.\left\lfloor\left(\frac{t}{500}\right)\right\rfloor\right)}$. 


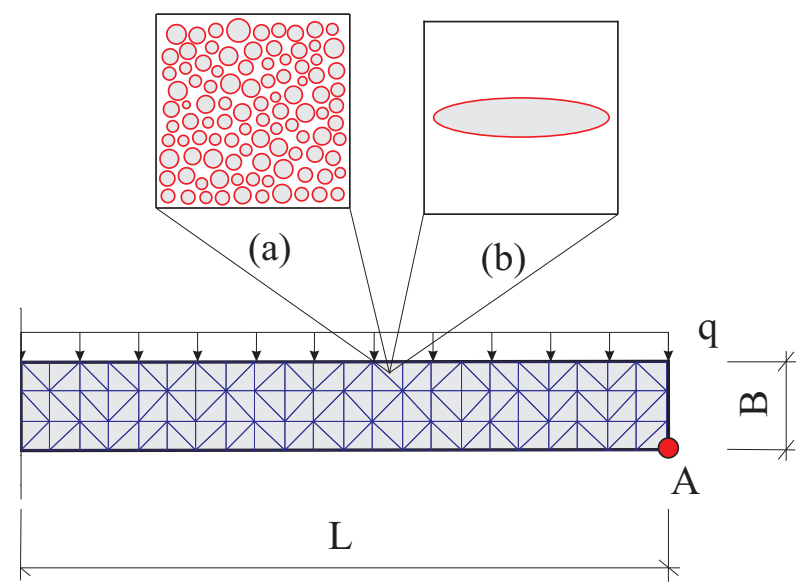

Figure 10: Two-scale analysis of a 2D beam structure: geometry and boundary conditions; a) and b) represent the different type of RVE related to the microstructure.

\subsection{Two scales analysis}

The purpose of this example is to compute the response of a beam composed of a linearly viscoelastic heterogeneous material. The geometry and boundary conditions of the problem considered are shown in figure 10 . The beam is subjected to a permanent loading $q=0.1 \mathrm{MPa} / \mathrm{m}$ on the upper surface. The dimensions of the beam are $L=10 \mathrm{~m}$ and $B=1 \mathrm{~m}$. The domain is meshed with triangular elements. The beam response will be calculated by the algorithm presented in section 3.4. As no analytical solution exists for such a problem, we have constructed a reference solution by applying a multilevel Finite Element Method $\left(F E^{2}\right)[4]$ where the stress-strain relationship is determined at each integration point of the macroscopic mesh through a local FEM computation. For comparison, the time step is the same for both methods. Two microstructures of RVE will be analyzed ((a) and (b) in figure 10), corresponding to those studied in the previous examples.

\subsubsection{RVE containing one elliptical inclusion}

In this case, the RVE contains an elliptical inclusion as in example 4.1.1. The time-dependent vertical displacement due to the permanent loading $q$ is computed for point A (see figure 10). Results are presented in figure 11. We define a relative error $e_{1}$ as 


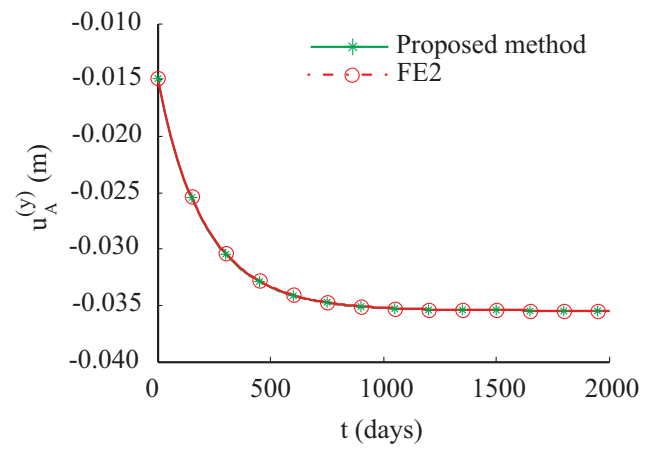

a)

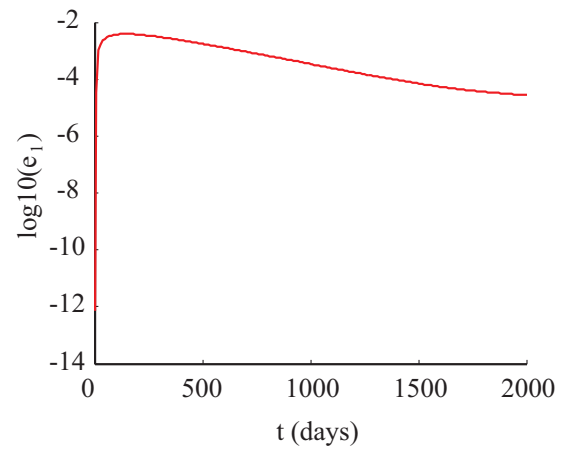

b)

Figure 11: Two-scale beam example with an RVE containing one inclusion: a) vertical displacement of a point in the structure; comparison between the proposed method and a reference $\left(\mathrm{FE}^{2}\right)$ solution; b) plot of the relative error $e_{1}(t)$ during the simulation

$$
e_{1}=\frac{\left|u_{A}^{(y)}(t)-u_{A, F E 2}^{(y)}(t)\right|}{\left|u_{A, F E 2}^{(y)}(t)\right|} .
$$

where $u_{A}^{(y)}$ and $u_{A, F E 2}^{(y)}$ are the vertical displacements of point A delivered by the proposed and $\mathrm{FE}^{2}$ methods, respectively. A macroscopic mesh with 300 triangular elements is used, and the macroscopic time step is $\bar{\Delta} t=20$ days. Results are provided in figure $11 \mathrm{~b}$ ), showing that the error remains relatively small during the whole simulation time.

Let $h$ be a characteristic element size of the macroscopic mesh. The convergence of the proposed method versus $h$ is analyzed. We depict, in figure $12 \mathrm{a}$ ), the vertical displacement $u_{A}^{(y)}(t)$ of point $\mathrm{A}$ for different mesh refinements. The reference solution is obtained with the proposed method and a very fine mesh $\left(h_{r e f}=1 / 100 \mathrm{~m}\right)$. The convergence is observed. To study the convergence of the solution with respect to $h$ and $\bar{\Delta} t$, we define the relative error $e_{2}(h, \bar{\Delta} t)$ as:

$$
e_{2}(h, \bar{\Delta} t)=\frac{\int_{0}^{T}\left|u_{A}^{(y)}(t)-u_{A, r e f}^{(y)}(t)\right| d t}{\int_{0}^{T}\left|u_{A, r e f}^{(y)}(t)\right| d t} .
$$

In figure $12 \mathrm{~b}), e_{2}(h)$ is plotted for a constant $\bar{\Delta} t=10$ days. The total simulation time is $T=2000$ days. The macroscopic mesh convergence is clearly observed. 


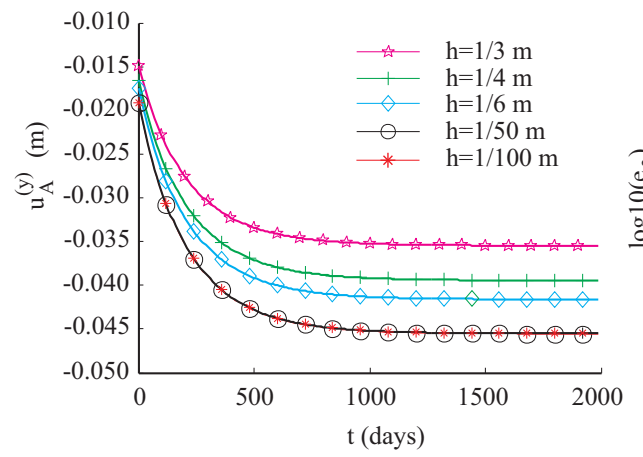

a)

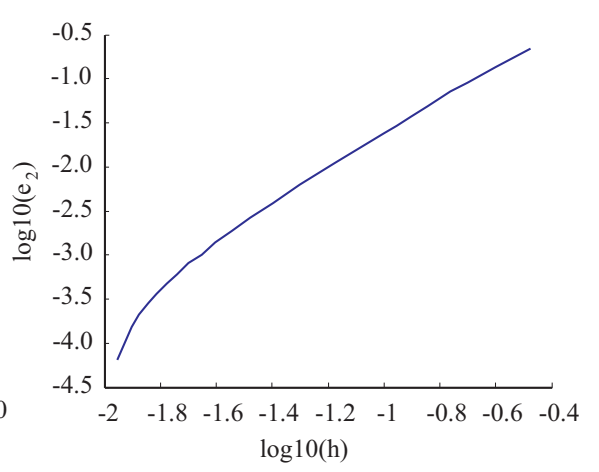

b)

Figure 12: Two-scale beam example with an RVE containing one inclusion: a) convergence of the solution (displacement of point $A$ in the structure) for different mesh sizes $h$; b) relative error $e_{2}$ with respect of the mesh size $h$ for a constant $\bar{\Delta} t$.

To study the convergence of the proposed method with respect to the macroscopic time step $\bar{\Delta} t$, we now take a constant $h$ and compute the solution and $e_{2}$ for different $\bar{\Delta} t$. Convergence results are provided in figure 13 with $h=1 / 4 \mathrm{~m}$ and $\bar{\Delta} t_{\text {ref }}=0.5$ days.

As expected, $F E^{2}$ calculations carried out to provide a reference solution are extremely time-consuming. For this reason, we did not examine the convergence of the solution with respect to $h$ and $\bar{\Delta} t$ for the $F E^{2}$ method.

In the following, we analyze the memory requirements of the proposed approach. In figure 14, the consumed memory of the two-scale simulation in question is plotted versus $\bar{\Delta} t$ for $\mathrm{FE}^{2}$ and the proposed method. The beam comprises 500 elements, and the RVE is meshed with 1264 elements. In the total memory, the main part is occupied by the strain and stress histories in all elements and by internal variables in the case of $F E^{2}$. The memory required to store the stiffness matrix is negligible in comparison. We observe that, beyond a given time step $\bar{\Delta} t$, the proposed method allows saving a lot of memory. For example, taking an error $e_{2} \leq e_{t o l}=10^{-6}$ corresponding to a time step $\bar{\Delta} t \geq 2.5$ (see figure $13 \mathrm{~b}$ )) is sufficient for the proposed approach to be advantageous over $F E^{2}$.

The comparison of the computation times is also shown in table 3 where $h$ is the mesh size at the macroscopic scale, $\mathrm{T}$ is the maximum simulation time, $T^{m i}$ is the time necessary for constructing the data base related to $\bar{\Gamma}_{i j k l}(t)$ from microscopic calculations on the RVE ( see section 3.4.1), $T^{m a}$ is the time of the macroscopic structure calculation. For the proposed method, 


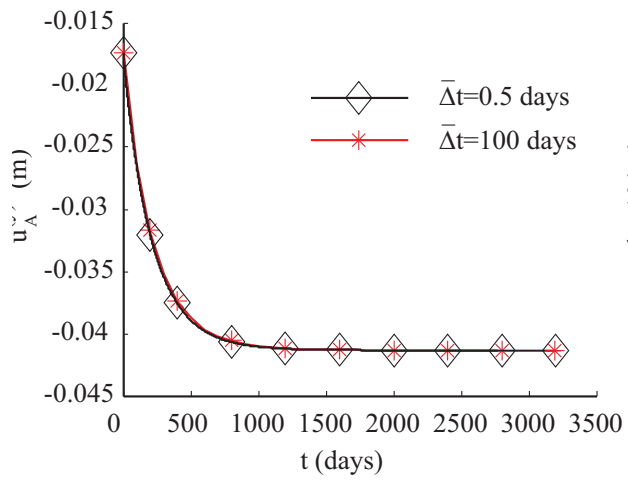

a)

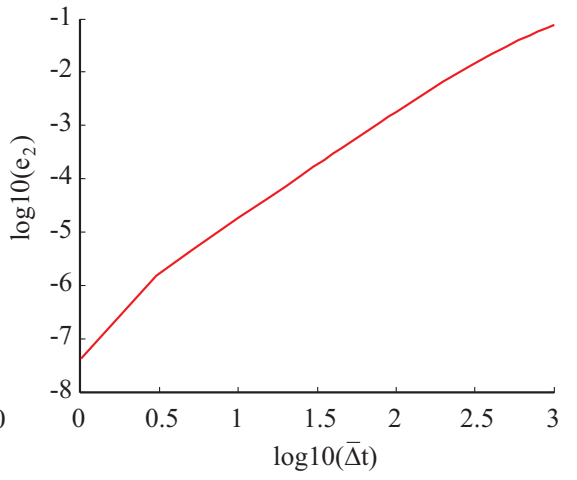

b)

Figure 13: Two-scale beam example with an RVE containing one inclusion: a) convergence of the solution (displacement of a point in the structure) for different macroscopic time steps $\bar{\Delta} t$; b) relative error $e_{2}$ with respect to $\bar{\Delta} t$ for a constant mesh size $h$.

the total computation time including preliminary calculations and structure calculation is given by $T^{t o t}=T^{m i}+T^{m a}$. For $\mathrm{FE}^{2}$ method, the computation time is denoted by $T_{F E^{2}}^{t o t}$. We can note that time saving ranges between 65 and 332 .

Table 3: Computation times of proposed method and $\mathrm{FE}^{2}$ for 2-D beam.

\begin{tabular}{|c|c|c|c|c|c|c|c|c|}
\hline Example & $\begin{array}{c}\bar{\Delta} t \\
(\mathrm{~d})\end{array}$ & $\begin{array}{c}h \\
(\mathrm{~m})\end{array}$ & $\begin{array}{c}T \\
(\mathrm{~d})\end{array}$ & $\begin{array}{c}T^{m i} \\
(\mathrm{~min})\end{array}$ & $\begin{array}{c}T^{m a} \\
(\mathrm{~min})\end{array}$ & $\begin{array}{c}T^{\text {tot }} \\
(\mathrm{min})\end{array}$ & $\begin{array}{c}T_{F E^{2}}^{\text {tot }} \\
(\mathrm{min})\end{array}$ & $\frac{T^{\text {tot }}}{T_{F E^{2}}}$ \\
\hline 4.2 .1 & 2.30 & 0.2500 & 3240 & 4.1 & 10.0 & 14.1 & 914 & 65.11 \\
\hline 4.2 .1 & 23.20 & 0.2500 & 3240 & 0.5 & 0.8 & 1.3 & 91 & 68.45 \\
\hline 4.2 .1 & 10.00 & 0.0625 & 2000 & 1.0 & 4.8 & 5.8 & 1406 & 243.79 \\
\hline 4.2 .1 & 10.00 & 0.0256 & 2000 & 1.0 & 19.6 & 20.6 & 6846 & 332.34 \\
\hline 4.2 .2 & 3.130 & 0.2500 & 3240 & 350.0 & 6.5 & 356.5 & 76430 & 214.41 \\
\hline 4.2 .2 & 31.26 & 0.2500 & 3240 & 35.7 & 0.6 & 36.3 & 7709 & 212.38 \\
\hline
\end{tabular}

\subsubsection{RVE containing 100 circular inclusions}

Here the same two-scale problem as in the foregoing example is analyzed but the RVE contains 100 circular inclusions. The beam is meshed with 


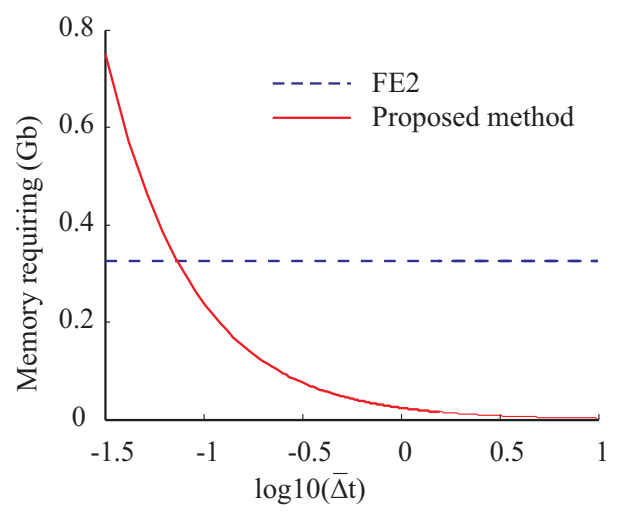

Figure 14: Two-scale beam example with an RVE containing one inclusion: memory required for the whole simulation versus macroscopic time step $\bar{\Delta} t$.

300 linear elements. The same parameters as in the previous example are adopted. Results are presented in figures 15, 16, 17 and 18. Though the microstructure is much more complex than in the previous example, very good accuracy and convergence are noticed. The same conclusions also hold concerning memory requirements.

\subsection{Three-dimensional dam}

In this example, a 3D dam model is studied. The objective is to determine the creep of the dam subjected to water pressure. The problem geometry is depicted in figure 19. The dam dimensions (see figure $19 \mathrm{~b}$ )) are such that $b_{1}=1.5 \mathrm{~m}, b_{2}=3 \mathrm{~m}, H=10 \mathrm{~m}$ and $l=l_{B M}=20 \mathrm{~m}$. The sides $B C D E$, $M N P Q$ and $C D P Q$ are blocked. The water pressure varies linearly from 0 at the top to $q g H$ at the bottom of the dam with $q=1000 \mathrm{~kg} / \mathrm{m}^{3}$ and $g$ being the acceleration of gravity. The water pressure is prescribed on the side $B C Q M$. Three different meshes are considered to study the convergence, containing 3370, 28181 and 435661 tetrahedra elements, respectively.

The material is heterogeneous and characterized by an RVE shown in figure 20. This RVE contains eight $1 / 8$ spheres located at the 8 corners and one sphere positioned at the center of the cube. The radius of the spheres is $0.4 L, L$ being the side length of the cube. The inclusion volume fraction is 0.5362. Both the matrix and inclusion materials are assumed to be isotropic. The matrix is viscoelastic with five Maxwell-elements and one elastic element (see figure 2). The inclusions are elastic. Their material parameters are provided in Table 4. A conforming mesh with 2561 linear tetrahedra is used 


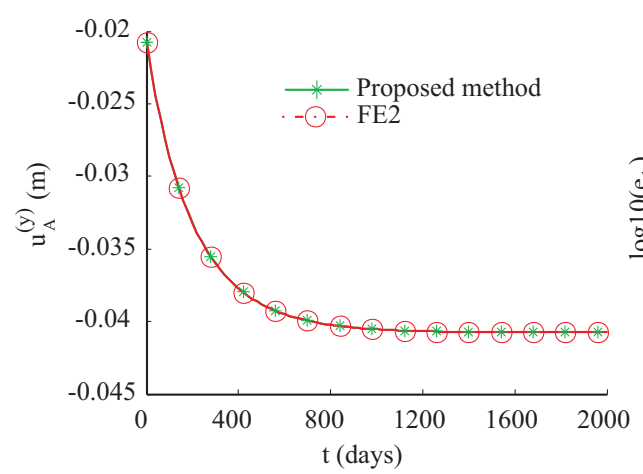

a)

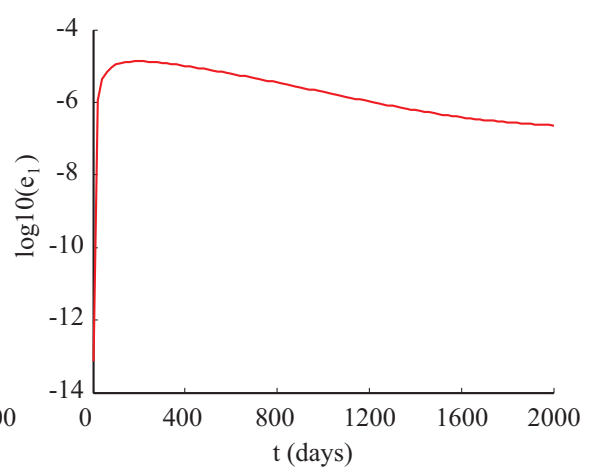

b)

Figure 15: Two-scale beam example with an RVE containing 100 inclusions: a) vertical displacement of a point in the structure; comparison between the proposed method and a reference $\left(\mathrm{FE}^{2}\right)$ solution; b) plot of the relative error $e_{1}(t)$ during the simulation

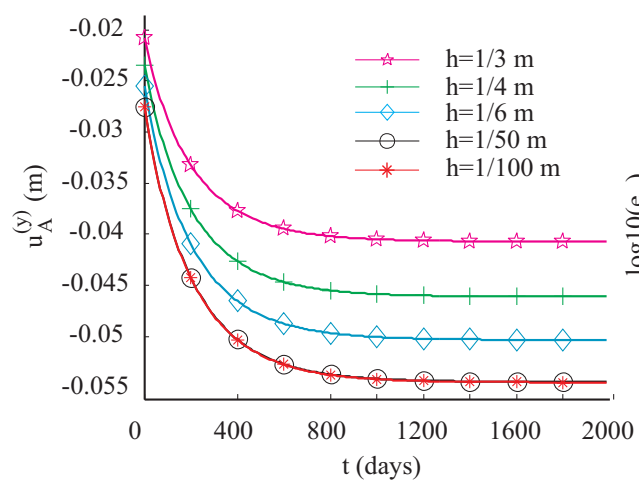

a)

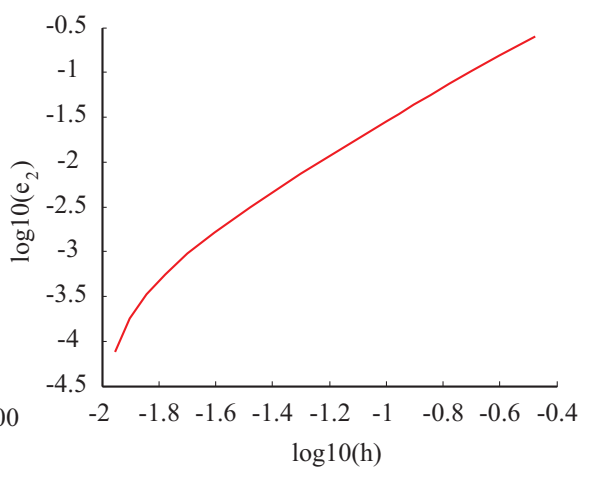

b)

Figure 16: Two-scale beam example with an RVE containing 100 inclusion: a) convergence of the solution (displacement of point $A$ in the structure) for different mesh sizes $h$; b) relative error $e_{2}$ with respect of the mesh size $h$ for a constant $\bar{\Delta} t$. 


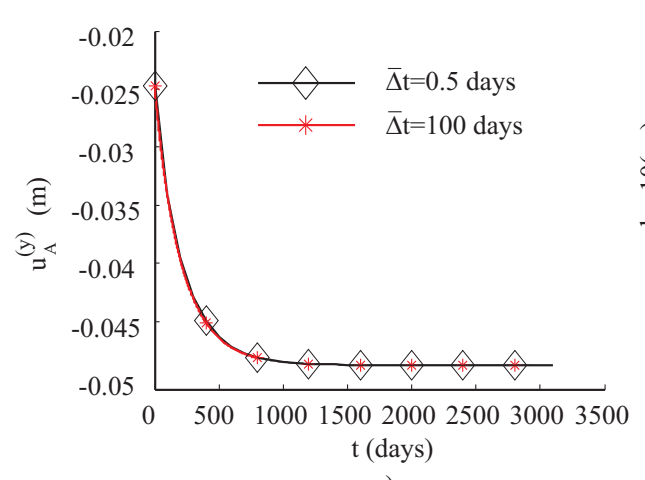

a)

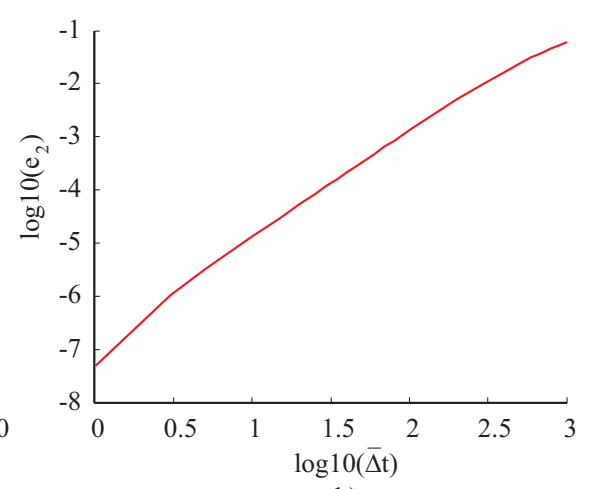

b)

Figure 17: Two-scale beam example with an RVE containing 100 inclusion: a) convergence of the solution (displacement of a point in the structure) for different macroscopic time steps $\bar{\Delta} t$; b) relative error $e_{2}$ with respect to $\bar{\Delta} t$ for a constant mesh size $h$.

Table 4: Material parameters of the phases in the RVE for the 3D example.

\begin{tabular}{llllll}
\hline Matrix parameters & & & & & \\
\hline$E_{\infty, m}$ (MPa.days) & 13909 & & & & \\
$\nu_{\infty, m}$ & 0.256 & & & & \\
$E_{m}^{e}$ (MPa.days) & 6930 & 9660 & 12750 & 18900 & 17310 \\
$\nu_{m}^{e}$ & 0.1 & 0.2 & 0.3 & 0.1 & 0.25 \\
$E_{m}^{v}$ (MPa.days) & 2012000 & 2555000 & 3489000 & 5030000 & 6577000 \\
$\nu_{m}^{v}$ & 0.1 & 0.2 & 0.3 & 0.1 & 0.25 \\
\hline Inclusion parameters & & & & & \\
\hline$E_{i}$ (MPa) & 1098400 & & & & \\
$\nu_{i}$ & 0.28 & & & & \\
\hline
\end{tabular}

for discretization of the RVE.

As in previous examples, we use the proposed multiscale procedure and the $F E^{2}$ method. We analyze the y-component of displacement vector for point A located at the middle of segment $B M$ in figure 19 . The corresponding results are provided in figure $21 \mathrm{a}$ ). A very good agreement is noticed between both methods. Figure $21 \mathrm{~b}$ ) shows relation between the relative error $e_{1}$ defined in (67) and the time steps $\bar{\Delta} t$. For this comparison, we have chosen the same time steps at the macro-scale and at the micro-scale.

The convergence of the solution versus the macroscopic time step is analyzed in figure 22. The reference solution is delivered by the present approach for a very small time step $\bar{\Delta} t_{r e f}=3$ days. 


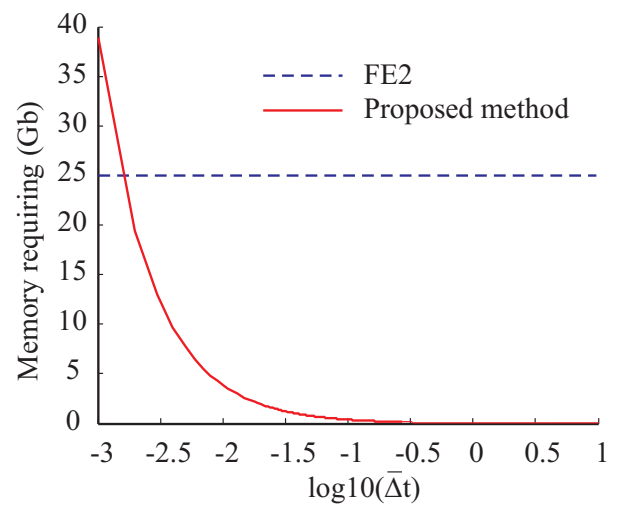

Figure 18: Two-scale beam example with an RVE containing one inclusion: memory required for the whole simulation versus macroscopic time step $\bar{\Delta} t$.
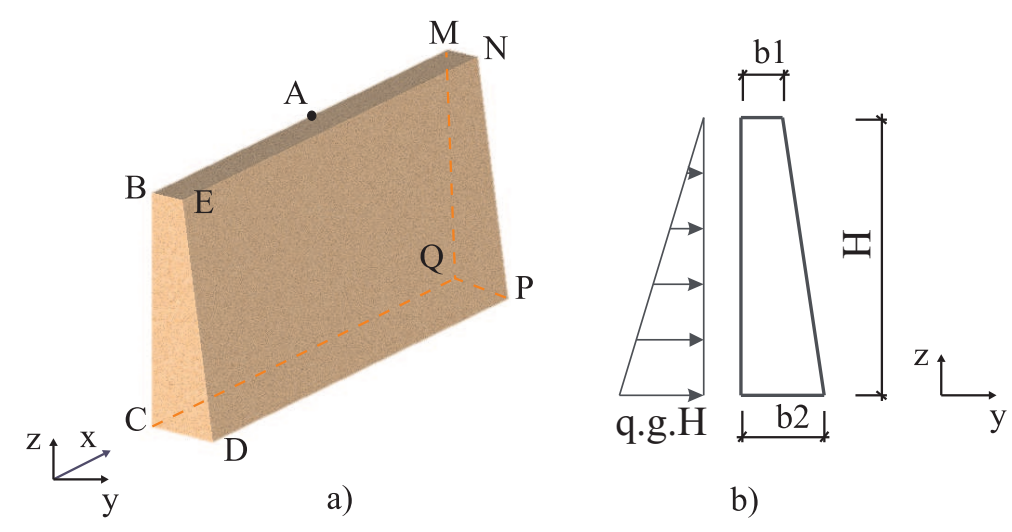

b)

Figure 19: a) Three dimensional dam b) Transversal section of the dam and water pressure distribution.

The convergence analysis with respect to the macroscopic mesh size $h$ is also carried out and illustrated in figure 23.

The memory requirements for both methods are presented in figure 24 . For $\log _{10}(\bar{\Delta} t) \geq 1$, i.e, $e_{2} \geq 10^{-6}$ in the figure $22 \mathrm{~b}$ ), the proposed method entails much less memory than $F E^{2}$. The comparison of the total computation times of both methods is shown in the table 5 where $N_{m i}$ and $N_{m a}$ are the numbers of elements of the meshes at the microscale and macroscale. As compared to $F E^{2}$, the proposed method gives a computational time saving ratio of the order of several hundreds. 


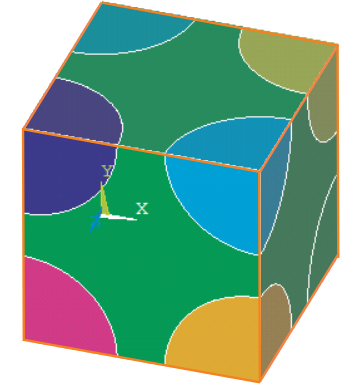

a)

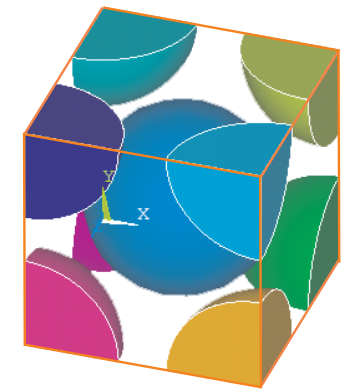

b)

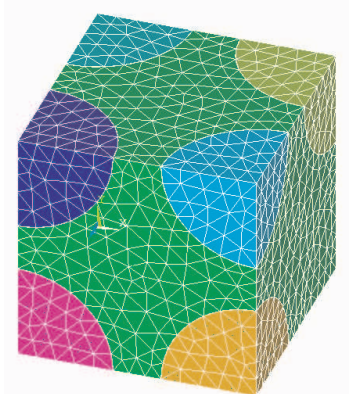

c)

Figure 20: a) RVE related to the dam material, containing one spherical inclusion and eight $1 / 8$ spherical inclusions; b) inclusions; c) 3D mesh.

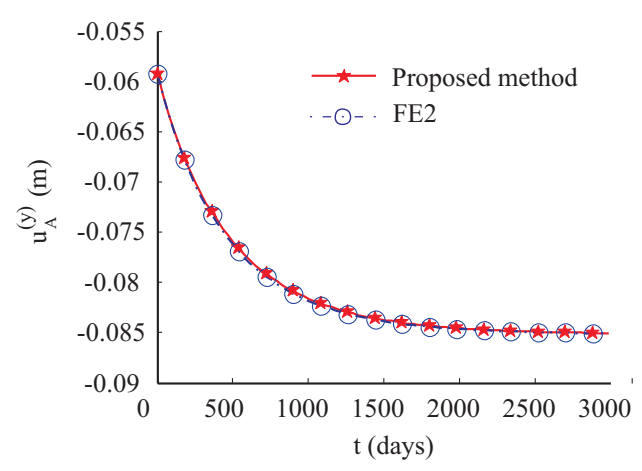

a)

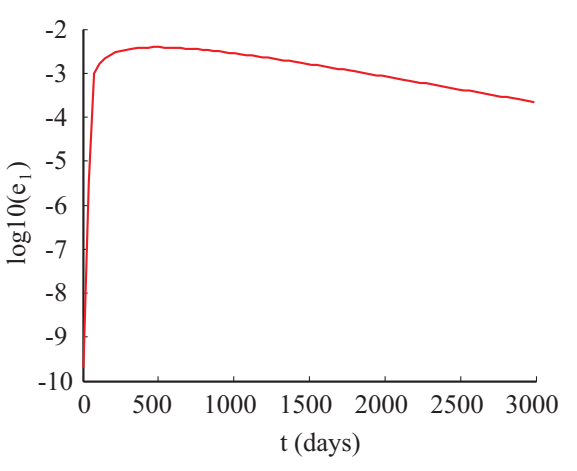

b)

Figure 21: Two-scale 3D dam example: a) y-displacement of a point in the structure; comparison between the proposed method and a reference $\left(\mathrm{FE}^{2}\right)$ solution; b) plot of the relative error $e_{1}(t)$ during the simulation. 


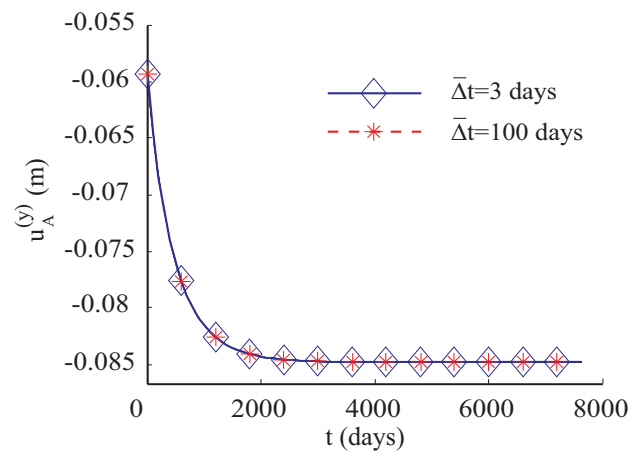

a)

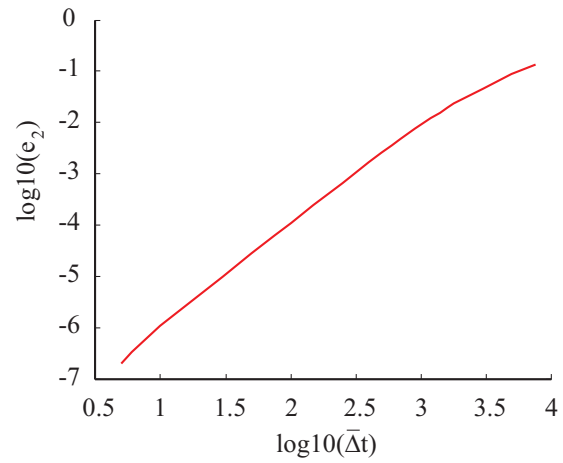

b)

Figure 22: Two-scale 3D dam example: a) convergence of the solution (displacement of point $A$ in the structure) for different $\bar{\Delta} t$ with $T=7650$ days; b) relative error $e_{2}$ with respect of $\bar{\Delta} t$.

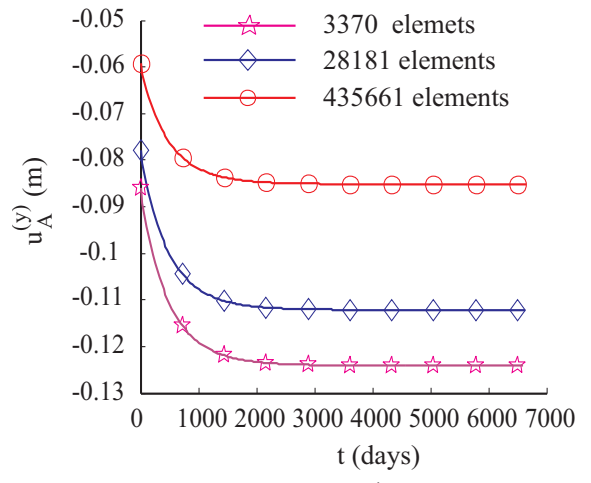

a)

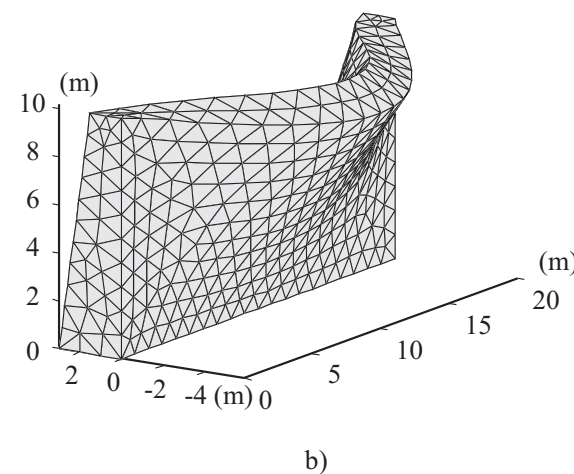

b)

Figure 23: Two-scale 3D dam example: a) convergence of the solution (displacement of a point in the structure) for different meshes with a constant microscopic time steps $\bar{\Delta} t=36$ days; b) Illustration of the deformed structure (magnified displacements). 


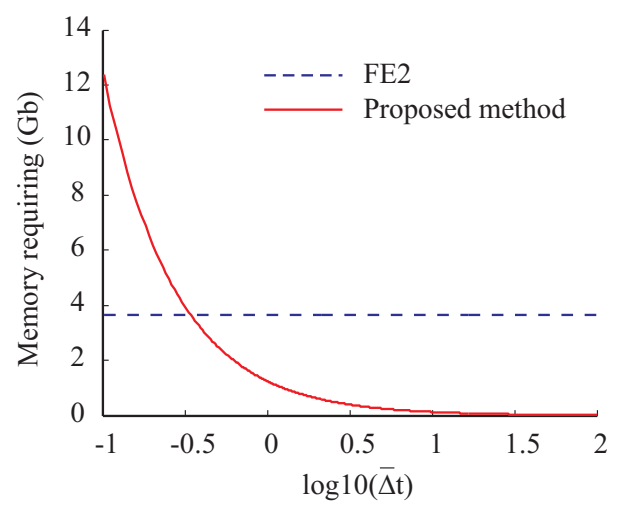

Figure 24: Relation between the memory requiring and the macroscopic time step $\bar{\Delta} t$.

Table 5: Computation time of proposed method and $\mathrm{FE}^{2}$ for 3-D dam.

\begin{tabular}{|c|c|c|c|c|c|c|c|c|}
\hline (d) & $N_{m a}$ & $N_{m i}$ & $\begin{array}{l}T \\
(\mathrm{~d})\end{array}$ & $\begin{array}{c}T^{m i} \\
(\min )\end{array}$ & $\begin{array}{c}T^{m a} \\
(\min )\end{array}$ & $\begin{array}{c}T^{t o t} \\
(\min )\end{array}$ & $\begin{array}{l}T_{F E^{2}}^{t o t} \\
(\mathrm{~min})\end{array}$ & $\frac{T^{t o t}}{T_{F E^{2}}^{t o t}}$ \\
\hline 36 & 3370 & 2561 & 7650 & 3.21 & 5.95 & 9.15 & 2840 & 310 \\
\hline 36 & 6865 & 8117 & 7650 & 20.58 & 10.25 & 30.83 & 6342 & 206 \\
\hline
\end{tabular}

\section{Conclusions}

We have presented in this work a multiscale method for computing the response of structures made of heterogeneous viscoelastic materials. Unlike the methods based on the Laplace transform, the present one operates directly in the time domain. It also avoids the use of macroscopic internal variables and nested numerical solvers at the micro and macro scales. The method comprises two steps. First, finite elements computations are carried out on an RVE model characterizing the microstructure. The components of the effective relaxation tensor are sampled for different snapshots in the time domain. In the second step where macroscopic structure calculations are performed, the effective constitutive law is expressed as a convolution product, involving the computed relaxation tensor, and an interpolation procedure is employed to numerically evaluate the convolution integral. The method elaborated decouples analyses at the different scales: once the data related to the effective tensor are computed and stored, structure calculations can 
be carried out without solving again the problem on the RVE. Finally, implementation of this method is simple, entailing only classical implicit time stepping algorithms. The different numerical examples studied have demonstrated the robustness, efficiency and accuracy of our method. As compared to direct multiscale employing nester solvers, computational gain ratios are of the order of several hundreds.

\section{Acknowledgements}

The support this work enjoys from EDF is gratefully acknowledged.

\section{Appendix : Relaxation tensor for the generalized Maxwell model}

Here, we expose a classical method for computing the generalized Maxwell relaxation moduli by using the Laplace transform. For the sake of simplicity, the method will be explained in the one-dimensional context. The system of equations associated with the generalized Maxwell model (figure 2) is as follows:

$$
\left\{\begin{array}{l}
\sigma_{\infty}(t)=E_{\infty} \varepsilon(t) \\
\sigma_{i}(t)=E_{i}\left(\varepsilon(t)-\alpha_{i}(t)\right)=\eta_{i} \dot{\alpha}_{i}, \\
\sigma(t)=\sigma_{\infty}(t)+\sum_{i=1}^{N} \sigma_{i}(t) .
\end{array}\right.
$$

In (69), $\sigma_{i}$ is the stress in element $i, \alpha_{i}$ is the strain related with damper $i, \sigma$ and $\varepsilon$ denotes the overall stress and strain of the system. The dot $\dot{x}$ refers to time-derivative of $x$. By applying the Laplace transform to (69), we obtain:

$$
\left\{\begin{array}{l}
\sigma_{\infty}^{*}(p)=E_{\infty} \varepsilon^{*}(p) \\
\sigma_{i}^{*}(p)=E_{i}\left(\varepsilon^{*}(p)-\alpha_{i}^{*}(p)\right)=\eta_{i} p \alpha_{i}^{*}(p) \\
\sigma^{*}(p)=\sigma_{\infty}^{*}(p)+\sum_{i=1}^{N} \sigma_{i}^{*}(p)
\end{array}\right.
$$

where $p$ is the complex frequency. From Equation $\left(70_{2}\right)$ we have:

$$
\alpha_{i}^{*}(p)=\frac{E_{i} \varepsilon^{*}(p)}{\eta_{i} p+E_{i}}
$$

and the total stress can be expressed by

$$
\sigma_{i}^{*}(p)=\frac{\eta_{i} p E_{i} \varepsilon^{*}(p)}{\eta_{i} p+E_{i}}
$$


Substituting $\sigma_{i}^{*}(p)$ and $\sigma_{\infty}^{*}(p)$ into Eq. $\left(70_{3}\right)$, we obtain

$$
\sigma^{*}(p)=\left(E_{\infty}+\sum_{i=1}^{N} \frac{\eta_{i} p E_{i}}{\eta_{i} p+E_{i}}\right) \varepsilon^{*}(p) .
$$

To obtain the relaxation function of the model, we apply a macroscopic strain $\varepsilon(t)=\varepsilon_{0} H(t)$. We have $\varepsilon^{*}(p)=L\{\varepsilon(t)\}=\varepsilon_{0} \frac{1}{p}$ with $L\{$.$\} being the$ Laplace transform operator. By Substituting $\varepsilon^{*}(p)$ into equation (73), yields

$$
\sigma^{*}(p)=\frac{1}{p}\left(E_{\infty}+\sum_{i=1}^{N} \frac{\eta_{i} p E_{i}}{\eta_{i} p+E_{i}}\right) \varepsilon_{0} .
$$

We obtain the total stress by applying the inverse Laplace transform to Eq. (74):

$$
\sigma(t)=G(t) \varepsilon_{0},
$$

where the relaxation modulus $G(t)$ is expressed by

$$
\begin{gathered}
G(t)=\mathcal{L}^{-1}\left\{\frac{1}{p}\left(E_{\infty}+\sum_{i=1}^{N} \frac{\eta_{i} p E_{i}}{\eta_{i} p+E_{i}}\right)\right\} \\
=E_{\infty}+\sum_{i=1}^{N} E_{i} \exp \left(-t / \tau_{i}\right)
\end{gathered}
$$

where $\tau_{i}$ is the relaxation time defined by $\tau_{i}=\eta_{i} / E_{i}$.

\section{References}

[1] S. Berbennia, V. Favier, X. Lemoine, M. Berveiller, Micromechanical modeling of the elastic-viscoplastic behavior of polycrystalline steels having different microstructures, Mater. Sci. Eng. A (2004), 372 (12):128-136.

[2] S. Beurthey, A. Zaoui, Structural morphology and relaxation spectra of viscoelastic heterogeneous materials, Eur. J. Mech. A-Solids (2000), 19:1-16.

[3] L. C. Brinson, W. S. Lin, Comparison of mircomechanics methods for effective properties of multiphase viscoelastic composites, Compos. Struct. (1998), 41:353-367.

[4] F. Feyel, Multiscale $\mathrm{FE}^{2}$ elastoviscoplastic analysis of composites structures, Comput. Mater. Sci. (1999), 16:344-354. 
[5] F.T. Fisher, L.C. Brinson, Viscoelastic interphases in polymer-matrix composites: theoretical models and finite-element analysis, Compos. Sci. Technol. (2001), 61:731-748.

[6] C. Friebel, I. Doghri, V. Legat, General mean-field homogenization schemes for viscoelastic composites containing multiple phases of coated inclusions, Int. J. Solids. Struct. (2006), 43:2513-2541.

[7] Z. Hashin, Viscoelastic behavior of heterogeneous media, J. Appl. Mech. Trans. ASME (1965), 32:630-636.

[8] Z. Hashin, Complex moduli of viscoelastic composites - I. General theory and application to particulate composites, Int. J. Solids Struct. (1970), 6:539-552.

[9] H. Hassanzadeh, M. Pooladi-Darvish, Comparion of different numerical Laplace inversion methods for engineering applications, Appl. Math. Comput. (2007), 189:1966-1981.

[10] M. Kaliske, H. Rothert, Formulation and implementation of threedimensional viscoelasticity at small and finite strains, Comput. Mech. (1997), 19(3):228-239.

[11] B. Kurnatowski, A. Matzenmiller, Finite element analysis of viscoelastic composite structures based on a micromechanical material model, Comput. Mater. Sci. (2008), 43:957-973.

[12] N. Lahellec, P. Suquet, Effective behavior of linear viscoelastic composites: A time-integration approach, Int. J. Solids. Struct. (2007), 44:507529.

[13] N. Laws, R. McLaughlin, Self-consistent estimates for the viscoelastic creep compliances of composite materials, Proc. R. Soc. Lond. A. (1978), 395:251-273.

[14] M. Lévesque, M. D. Gilchrist, N. Bouleau, K. Derrien, D. Baptiste, Numerical inversion of the Laplace-Carson transform applied to homogenization of randomly reinforced linear viscoelastic media, Comput. Mech. (2007), 40:771-789. 
[15] C. Maeau, V. Favier, M. Berveiller, Micromechanical modeling coupling time-independent and time-dependent behaviors for heterogeneous materials, Int. J. Solids. Struct. (2009), 46:223-237.

[16] P.S. Mangat, M.M. Azari, A theory of the creep of steel fibre reinforced cement matrices under compression, J. Mater. Sci. (1985), 20:1119-1133.

[17] A. Matzenmiller, S. Gerlach, Micromechanical modeling of viscoelastic composites with compliant fiber-matrix bonding, Comput. Mater. Sci (2003), 29(3):283-300.

[18] V.F. Pasa Dutra, S. Maghous, A. Campos Filho, A.R Pacheco, A micromechanical approach to elastic and viscoelastic properties of fiber reinforced concrete, Cem. Concr. Res. (2010), 40:460-472.

[19] P. Ponte Castañeda, New variational principles in plasticity and their application to composite materials, J. Mech. Phys. Solids (1992), 40:17571788.

[20] J. M. Ricaud, R. Masson, Effective properties of linear viscoelastic heterogeneous media: Internal variables formuation and extension to ageing behaviours, Int. J. Solids. Struct. (2009), 46:1599-1606.

[21] H. Sabar, M. Berveiller, V. Favier, S. Berbenni, A new class of micromacro models for elastic-viscoplastic heterogeneous materials, Int. J. Solids. Struct (2002), 39(12):2357-3276.

[22] J.C. Simo, T.J.R. Hughes, Computational Inelasticity, Springer (1998).

[23] P. Spanne, J.F. Thovert, C.J. Jacquin, Synchrotron computed microtomography of porous media - topology and transports, Phys. Rev. Lett (1994), 73(14):2001-2004.

[24] R. Taylor, K. Pister, G. Goudreau, Thermomechanical analysis of viscoelastic solids, Int. J. Num. Meth. Eng. (1970), 2:45-59.

[25] P. A. Turner and C. N. Tomé, Self-consistent modeling of visco-elastic polycrystals: Application to irradiation creep and growth, J. Mech. Phys. Solids (1993), 41(7):1191-1211.

[26] Y.M. Wang, G.J. Weng, The influence of inclusions shape on the overall viscoelastic behavior of composites, J. Appl. Mech (1992), 59:510-518. 
[27] W.T. Weeks, Numerical inversion of Laplace transforms using Laguerre functions, J. ACM (1966), 13(3):419-429.

[28] J. Yvonnet, D. Gonzalez, Q.-C. He, Numerically explicit potentials for the homogenization of nonlinear elastic heterogeneous materials, Comput. Meth. Appl. Mech. Eng. (2009), 198:2723-2737.

[29] J. Yvonnet, Q.-C. He, The Reduced Model Multiscale Method (R3M) for the nonlinear homogenization of hyperelastic media at finite strains, J. Comput. Phys. (2007) 223 : 341-368. 